\title{
Convergence Toward a Periodically Rotating One-Point Cluster in the Kinetic Thermodynamic Cucker-Smale Model
}

\author{
Linglong $\mathrm{Du}^{1, *}$ and Seung-Yeal $\mathrm{Ha}^{2}$ \\ ${ }^{1}$ Department of Applied Mathematics, Donghua University, Shanghai, \\ P.R. China. \\ ${ }^{2}$ Department of Mathematical Sciences and Research Institute of \\ Mathematics, Seoul National University, Seoul 08826, Republic of Korea.
}

Received 17 October 2021; Accepted 2 November 2021

\begin{abstract}
We study a relaxation dynamics of the kinetic thermodynamic Cucker-Smale (TCS) model under the effect of potential force field. For this, we present a sufficient framework on the emergence of a periodically rotating onepoint cluster to the TCS model with a harmonic potential force. In the presence of external potential force, large-time behavior of the kinetic TCS model is completely different from that of the kinetic TCS model without an external force. For the relaxation toward the periodic motion in thermo-mechanical observables, we use dynamical systems theory such as the Lyapunov functional approach, method of characteristics and continuity argument. First, we derive the exponential decay estimate for the Lyapunov functionals of thermo-mechanical observables for the kinetic density $f$ with a compact support. Second, we show that the supports of $f$ in $(x, v, \theta)$ shrink to a periodically rotating one-point cluster exponentially fast. Our sufficient framework is formulated in terms of initial configuration, coupling strengths and communication weight functions.
\end{abstract}

AMS subject classifications: 35B35, 74A15, 93C20

Key words: One-point cluster, harmonic potential field, thermodynamic Cucker-Smale ensemble, kinetic model.

\footnotetext{
*Corresponding author. Email addresses: matdl@dhu.edu.cn (L. Du), syha@snu. ac.kr (S.-Y. Ha)
} 


\section{Introduction}

The purpose of this paper is to continue previous studies in [7] on the formation of one-point cluster in the thermodynamic Cucker-Smale ensemble under the effect of a harmonic potential field. Collective behaviors of many-body systems are often observed in nature, to name a few, aggregation of bacteria [25], flashing of fireflies [3,27], flocking of birds [26], schooling of fish [11,22,24] and synchronous firing of pacemaker cells [21], etc. For the brief introduction of the subject, we refer to survey articles $[1,2,20]$. To provide a realistic model for the collective behaviors of active particles, we need to introduce a dynamical system taking into account not only interactions between mechanical observables (position and velocity) but also extra interactions due to the internal observables such as temperature and spins, etc. As a first step toward the realistic flocking modeling of active particles, Ha and Ruggeri introduced a generalized CuckerSmale model [18] for thermo-mechanical ensemble, so called the thermodynamic Cucker-Smale model. In this work, we further investigate the effect of an external potential force field on the emergent dynamics of the thermodynamic CuckerSmale ensemble.

Let $x_{\alpha}, v_{\alpha}$ and $\theta_{\alpha}$ be the position, velocity and temperature of the $\alpha$-th TCS particle, respectively. Then, their temporal dynamics is governed by the Cauchy problem to the following (particle) TCS model:

$$
\left\{\begin{aligned}
\frac{d x_{\alpha}}{d t}=v_{\alpha}, \quad t>0, \quad \alpha \in[N]:=\{1, \ldots, N\} & \frac{d v_{\alpha}}{d t}=\frac{\kappa_{1}}{N} \sum_{\beta=1}^{N} \psi\left(\left|x_{\alpha}-x_{\beta}\right|\right)\left(\frac{v_{\beta}-\bar{v}}{\theta_{\beta}}-\frac{v_{\alpha}-\bar{v}}{\theta_{\alpha}}\right)-\nabla_{x_{\alpha}} U\left(x_{\alpha}\right), \\
\frac{d}{d t}\left(\theta_{\alpha}+\frac{1}{2}\left|v_{\alpha}\right|^{2}\right)= & \frac{\kappa_{2}}{N} \sum_{\beta=1}^{N} \zeta\left(\left|x_{\alpha}-x_{\beta}\right|\right)\left(\frac{1}{\theta_{\alpha}}-\frac{1}{\theta_{\beta}}\right) \\
& +\frac{\kappa_{1}}{N} \sum_{\beta=1}^{N} \psi\left(\left|x_{\alpha}-x_{\beta}\right|\right)\left(\frac{v_{\beta}-\bar{v}}{\theta_{\beta}}-\frac{v_{\alpha}-\bar{v}}{\theta_{\alpha}}\right) \cdot \bar{v},
\end{aligned}\right.
$$

subject to initial data

$$
\left(x_{\alpha}, v_{\alpha}, \theta_{\alpha}\right)(0)=\left(x_{\alpha}^{0}, v_{\alpha}^{0}, \theta_{\alpha}^{0}\right),
$$

where $\kappa_{1}$ and $\kappa_{2}$ denote nonnegative coupling strengths, and the ensemble averages $(\bar{x}, \overline{\mathcal{v}})$ are given as follows:

$$
\bar{x}:=\frac{1}{N} \sum_{\alpha=1}^{N} x_{\alpha}, \quad \bar{v}:=\frac{1}{N} \sum_{\alpha=1}^{N} v_{\alpha} .
$$


Here $|\cdot|$ denotes the standard $\ell^{2}$-norm in $\mathbb{R}^{d}$. We assume that the communication weight functions $\psi$ and $\zeta$ are nonnegative and Lipschitz continuous functions such that

$$
\left\{\begin{array}{lll}
0 \leq \psi(r) \leq \psi(0)=1, & 0 \leq \zeta(r) \leq \zeta(0)=1, & r \geq 0 \\
\left(\psi\left(r_{2}\right)-\psi\left(r_{1}\right)\right)\left(r_{2}-r_{1}\right) \leq 0, & \left(\zeta\left(r_{2}\right)-\zeta\left(r_{1}\right)\right)\left(r_{2}-r_{1}\right) \leq 0, & r_{1}, r_{2} \geq 0
\end{array}\right.
$$

In this paper, we consider a mesoscopic regime in which the number of particles $N$ is sufficiently large so that the dynamics of (1.1) can be well approximated by one-particle distribution function. To set the stage, we also assume that $U(x)=$ $|x|^{2} / 2$ as a potential for an external force. In this regime, the dynamics of the TCS model with $N \gg 1$ can be effectively approximated by the corresponding mean-field kinetic model. More precisely, let $f=f(t, x, v, \theta)$ be the one-particle distribution function at position $x$, with velocity $v$, temperature $\theta$, and at time $t \geq 0$. For simplicity, we set

$$
\Omega:=\mathbb{R}^{d} \times \mathbb{R}^{d} \times \mathbb{R}_{+}, \quad z:=(x, v, \theta) \in \Omega .
$$

Then, the mean-field kinetic equation can be formally derived using the BBGKY hierarchy argument in [19] and rigorously justified using the particle-in-cell method as in [17]. In this situation, the dynamics of the kinetic density $f$ with the unit mass is governed by the following Cauchy problem:

$$
\left\{\begin{aligned}
& \partial_{t} f+\nabla_{x} \cdot(v f)+\nabla_{v} \cdot(\mathcal{F}[f] f)+\partial_{\theta}(\mathcal{G}[f] f)=0, \quad t>0, \quad z \in \Omega, \\
& \mathcal{F}[f]:=-\kappa_{1} \int_{\Omega} \psi\left(\left|x-x_{*}\right|\right)\left(\frac{v-v_{c}}{\theta}-\frac{v_{*}-v_{\mathcal{C}}}{\theta_{*}}\right) f_{*} d z_{*}-x, \\
& \mathcal{G}[f]:= \kappa_{1} \int_{\Omega} \psi\left(\left|x-x_{*}\right|\right)\left(v-v_{\mathcal{C}}\right) \cdot\left(\frac{v-v_{c}}{\theta}-\frac{v_{*}-v_{C}}{\theta_{*}}\right) f_{*} d z_{*} \\
&+\kappa_{2} \int_{\Omega} \zeta\left(\left|x-x_{*}\right|\right)\left(\frac{1}{\theta}-\frac{1}{\theta_{*}}\right) f_{*} d z_{*}+x \cdot v, \\
& f(0, z)= f^{0}(z), \quad\left\|f^{0}\right\|_{L^{1}}=1,
\end{aligned}\right.
$$

where we used a handy notation $f=f(t, z), f_{*}=f\left(t, z_{*}\right)$ and $v_{c}:=\int_{\Omega} v f d z$.

The kinetic model (1.3) bridges particle description and hydrodynamic description on the emergent dynamics of the CS ensemble as an intermediate description, and it has been extensively investigated by many authors from various point of views, e.g., classical solutions $[6,19]$, measure-valued solutions and mean-field limit $[4,8-10,12,14,16,17]$, emergent dynamics $[7,15,18]$. We also refer 
to $[5,13,23]$ for the hydrodynamic CS model with an external convex potential force. In this paper, we address the following simple question:

(Q): Under what conditions on initial configuration and system parameters, can we rigorously verify the formation of periodically rotating one-point cluster for the kinetic model (1.3)?

The purpose of this paper is to exploit the above question from the analytic point of views, i.e., we derive a sufficient framework leading to the formation of one-point cluster to the kinetic model (1.3). In the absence of external forcing, i.e., $U \equiv 0$, the Cauchy problem admits the conservation of linear momentum so that state tends to a translational motion with a fixed velocity (initial average velocity $\left.v_{\mathcal{C}}(0)\right)$. In this case, many researchers showed the emergence of local and global flocking behaviors by lifting the results for the particle model to the corresponding kinetic model using dynamical systems theory such as Lyapunov functional approach, method of characteristics and continuity argument to derive the longtime flocking behaviors. In contrast, when the CS ensemble is under the effect of an external harmonic potential field $-\nabla_{x}\left(|x|^{2} / 2\right)=-x$, this momentum conservation law breaks down. In fact, the average mechanical observables $\left(x_{c}, v_{c}\right)$ are not translational motion any more, but in fact, they satisfy the harmonic oscillator motion (periodic motion). See Lemma 2.1.

The novelty of this paper is to provide an explicit framework in which mechanical observables relax to the harmonic oscillator motion, while the temperature field relaxes to the average temperature which is periodic too. More specifically, our main results are concerned with uniform bounds and decay properties of diameters of thermo-mechanical observables associated with the compactly supported density $f$ for (1.3) under suitable framework in terms of initial configuration and system parameters. We list the sufficient framework leading to the asymptotic convergence toward periodic motion in position-velocity and relaxation of temperature field toward the average temperature to the kinetic TCS model (1.3) in Section 3.1. Let $\Omega_{\theta}(f, 0)$ be the projected support in temperature variable (see (1.5)). If $\min \Omega_{\theta}(f, 0)$ is positive, the local existence of a solution will be guaranteed by the continuity argument. To ensure a global existence of a solution, we have to guarantee that the temperature support has a positive lower bound for all time $t$. For the well-prepared initial configuration, suitably selected communication functions and coupling strengths ((3.2), (3.4) and (3.6)), we make an ansatz that the temperature support has a positive lower bound in a small-time interval $[0, \tau]$ (see (3.3)). We also assume that the projected support of $f$ in position and velocity variables are bounded in another small-time interval $\left[0, \tau^{*}\right] \subseteq[0, \tau]$ (see (3.5)). Then we derive an exponential decay result for the functional measuring variances of the position and velocity (see Proposition 4.1). We use this result 
and continuity argument to conclude the concentration of mechanical variables in $[0, \tau]$ (see Proposition 4.2), and close the ansatz (3.5). To derive the concentration of temperature variable (see Proposition 5.2) and close the ansatz (3.3), an exponential decay result for the functional measuring the variance of the temperature is needed (see Proposition 5.1). Finally, we extend all the results in $[0, \tau]$ to $[0, \infty)$ (see Theorem 3.1) using the continuity argument and exponential decay properties.

The rest of this paper is organized as follows. In Section 2, we briefly discuss mean-field limit from the particle TCS model (1.1) to the kinetic TCS model (1.3) in the presence of harmonic potential field, and then we study a priori estimates for the kinetic model (1.3). In Section 3, we briefly list a sufficient framework leading to the periodically rotating one-point cluster and describe our main result without a proof. In Section 4 and Section 5, we prove the pointwise exponential concentration properties for the thermo-mechanical observables for the compactly supported density $f$. In Section 6, we provide a proof of main result. Finally, Section 7 is devoted to a brief summary of this paper.

Gallery of Notation: We set

$$
\begin{array}{llll}
X=\left(x_{1}, \cdots, x_{N}\right), & V=\left(v_{1}, \cdots, v_{N}\right), & \Theta=\left(\theta_{1}, \cdots, \theta_{N}\right), & z=(x, v, \theta), \\
Z=(X, V, \Theta), & \Omega:=\mathbb{R}^{2 d} \times \mathbb{R}_{+}, & \theta_{m}^{0}=\min _{\alpha} \theta_{\alpha}^{0}, & \theta_{M}^{0}=\max _{\alpha} \theta_{\alpha}^{0}, \\
d[X]=\max _{\alpha, \beta}\left|x_{\alpha}-x_{\beta}\right|, & d[V]=\max _{\alpha, \beta}\left|v_{\alpha}-v_{\beta}\right|, & d[\Theta]=\max _{\alpha, \beta}\left|\theta_{\alpha}-\theta_{\beta}\right| . &
\end{array}
$$

Let $f=f(t, z)$ be a one-particle distribution function at $z$ and at time $t$ with unit mass. Then, we set

$$
x_{c}:=\int_{\Omega} x f d z, \quad v_{c}:=\int_{\Omega} v f d z, \quad \theta_{c}:=\int_{\Omega} \theta f d z,
$$

and define diameter functions in $x, v$ and $\theta$

$$
\begin{aligned}
& d_{x}[f]:=\max \left\{\left|x-x_{*}\right|:(x, v, \theta), \quad\left(x_{*}, v_{*}, \theta_{*}\right) \in \operatorname{spt}(f)\right\}, \\
& d_{v}[f]:=\max \left\{\left|v-v_{*}\right|:(x, v, \theta),\left(x_{*}, v_{*}, \theta_{*}\right) \in \operatorname{spt}(f)\right\}, \\
& d_{\theta}[f]:=\max \left\{\left|\theta-\theta_{*}\right|:(x, v, \theta),\left(x_{*}, v_{*}, \theta_{*}\right) \in \operatorname{spt}(f)\right\},
\end{aligned}
$$

and projected supports:

$$
\begin{aligned}
& \Omega_{x}(f, t):=\overline{\left\{x \in \mathbb{R}^{d} \mid f(t, x, v, \theta) \neq 0\right\}}, \\
& \Omega_{v}(f, t):=\overline{\left\{v \in \mathbb{R}^{d} \mid f(t, x, v, \theta) \neq 0\right\}}, \\
& \Omega_{\theta}(f, t):=\overline{\left\{\theta \in \mathbb{R}_{+} \mid f(t, x, v, \theta) \neq 0\right\}} .
\end{aligned}
$$

Let $B_{R}(z)$ be the open ball with center $z$ with radius $R,|\cdot|_{p}$ be the $\ell^{p}$-norm in $\mathbb{R}^{d}$. 


\section{Preliminaries}

In this section, we briefly review the mean-field limit from the TCS model (1.1) to the corresponding kinetic TCS model (1.3) in the presence of a harmonic potential field, and present basic estimates for the kinetic TCS model.

\subsection{The TCS model}

Here, we recall several basic properties of the TCS model and briefly discuss the mean-field limit from the TCS model to the kinetic TCS model following the systematic procedure in [14]. For a given particle configuration $\left\{\left(x_{\alpha}, v_{\alpha}, T_{\alpha}\right)\right\}$, we set

$$
\mathcal{E}:=\sum_{\alpha=1}^{n}\left(T_{\alpha}+\frac{1}{2}\left|v_{\alpha}\right|^{2}\right)
$$

As in [7], one has the temporal evolution of the above functional.

Lemma 2.1 ([7]). For $\tau \in(0, \infty]$, let $\left\{\left(x_{\alpha}, v_{\alpha}, \theta_{\alpha}\right)\right\}$ be a smooth solution to (1.1) in a timeinterval $[0, \tau)$ with the initial data satisfying

$$
|\bar{x}(0)|<\infty, \quad|\bar{v}(0)|<\infty \text { and } \mathcal{E}(0)<\infty .
$$

Then, the following estimates hold:

1. The averaged position $\bar{x}$ and velocity $\bar{v}$ in (1.2) take the following explicit formulas:

$$
\left\{\begin{array}{l}
\bar{x}(t)=\bar{x}(0) \cos t+\bar{v}(0) \sin t, \quad t \in[0, \tau), \\
\bar{v}(t)=-\bar{x}(0) \sin t+\bar{v}(0) \cos t
\end{array}\right.
$$

2. The total energy satisfies

$$
\mathcal{E}(t)=\mathcal{E}(0), \quad t \in(0, \tau)
$$

Remark 2.1. Note that if we assume $\bar{x}(0)=0$ and $\bar{v}(0)=0$, one has

$$
\bar{x}(t)=0, \quad \bar{v}(t)=0, \quad t \geq 0 .
$$

Next, we discuss the mean-field limit of system (1.1). Most analysis can be treated exactly in a similar manner as in [14] except for additional terms due to the external potential force field. Since our main concern is not a mean-field limit here, we are not going to delve into details of the mean-field limit, but we instead 
delineate procedures step by step following [14] without excessive details. For the model (1.1), note that the temperature field is coupled with velocity field so that emergent analysis is pretty messy. Although the mean-field limit can be done for the full model, for the simplicity of presentation, we will consider the simplified TCS model in a harmonic potential field by setting the center of mass to zero. In this setting, the temporal evolution of the temperature field can be completely decoupled from that of velocity field

$$
\left\{\begin{array}{l}
\frac{d x_{\alpha}}{d t}=v_{\alpha}, \quad t>0, \quad \alpha \in[N], \\
\frac{d v_{\alpha}}{d t}=\frac{\kappa_{1}}{N} \sum_{\beta=1}^{N} \psi_{\alpha \beta}\left(\frac{v_{\beta}}{\theta_{\beta}}-\frac{v_{\alpha}}{\theta_{\alpha}}\right)-x_{\alpha}, \\
\frac{d \theta_{\alpha}}{d t}=\frac{\kappa_{2}}{N} \sum_{\beta=1}^{N} \zeta_{\alpha \beta}\left(\frac{1}{\theta_{\alpha}}-\frac{1}{\theta_{\beta}}\right), \\
\bar{x}(t)=0, \quad \bar{v}(t)=0 .
\end{array}\right.
$$

Then, the mean-field limit from the simplified TCS model (2.1) to the corresponding kinetic model

$$
\left\{\begin{array}{l}
\partial_{t} f+\nabla_{x} \cdot(v f)+\nabla_{v} \cdot(\mathcal{F}[f] f)+\partial_{\theta}(\mathcal{G}[f] f)=0, \quad t>0, \quad z \in \Omega, \\
\mathcal{F}[f]:=-\kappa_{1} \int_{\Omega} \psi\left(\left|x-x_{*}\right|\right)\left(\frac{v}{\theta}-\frac{v_{*}}{\theta_{*}}\right) f_{*} d z_{*}-x, \\
\mathcal{G}[f]:=\kappa_{2} \int_{\Omega} \zeta\left(\left|x-x_{*}\right|\right)\left(\frac{1}{\theta}-\frac{1}{\theta_{*}}\right) f_{*} d z_{*}, \\
\int_{\Omega} x f d z=\int_{\Omega} v f d z=0, \quad\left\|f^{0}\right\|_{L^{1}}=1
\end{array}\right.
$$

can be performed in the following three steps:

Step A. (Exponential flocking). For positive constants $\Gamma>0$ and $\kappa_{1}>0$, we define a set of admissible initial configurations

$$
\begin{aligned}
\mathcal{A}\left(\Gamma, \kappa_{1}\right):=\left\{Z^{0} \in \mathbb{R}^{(2 d+1) N}:\left|X^{0}\right| \leq \Gamma,\left|V^{0}\right| \leq \Gamma,\right. \\
\left.\frac{\sqrt{8\left(\theta_{m}^{0}\right)^{2}+2\left(d\left(\Theta^{0}\right)^{2}\right.}}{\left(\theta_{m}^{0}\right)^{2}} \leq \frac{1}{\kappa_{1}}, 0<\frac{\psi(3 \sqrt{2} \Gamma)}{\theta_{M}^{0}}-\frac{d\left(\Theta^{0}\right)}{2\left(\theta_{m}^{0}\right)^{2}}<\frac{1}{\kappa_{1}}\right\},
\end{aligned}
$$

where $|\cdot|$ is the standard $\ell^{2}$-norm in $\mathbb{R}^{d}$. 
Let $Z$ be a global solution to (2.1) with the initial data $Z^{0} \in \mathcal{A}\left(\Gamma, \kappa_{1}\right)$. Then one-point cluster emerges exponentially fast, which can be proved by the same arguments in [7]

$$
\begin{aligned}
& d[X(t)] \leq 4 \sqrt{\left|X^{0}\right|^{2}+\left|V^{0}\right|^{2}} e^{-\frac{1}{12} \kappa_{1} v t,} \\
& d[V(t)] \leq 4 \sqrt{\left|X^{0}\right|^{2}+\left|V^{0}\right|^{2}} e^{-\frac{1}{12} \kappa_{1} v t}, \\
& d[\Theta(t)] \leq \sqrt{2} d\left(\Theta^{0}\right) \exp \left[-\kappa_{2} \frac{\zeta(3 \sqrt{2} \Gamma)}{\left(\theta_{M}^{0}\right)^{2}} t\right] \text { as } t \rightarrow \infty,
\end{aligned}
$$

where

$$
v:=\frac{2 \psi(3 \sqrt{2} \Gamma)}{\theta_{M}^{0}}-\frac{d\left(\Theta^{0}\right)}{\left(\theta_{m}^{0}\right)^{2}} .
$$

The exponential decay estimates (2.3) will be used to verify the existence of measure-values solutions to the kinetic TCS model in the whole-time interval (see Step C).

Step B. (Uniform-in-time stability). For $p, q \in[1, \infty)$, let $Z$ and $\tilde{Z}$ be two global solutions to (2.1) with the initial data $Z^{0}$ and $\tilde{Z}^{0}$

$$
Z^{0} \in \mathcal{A}\left(\Gamma, \kappa_{1}\right), \quad \tilde{Z}^{0} \in \mathcal{A}\left(\Gamma, \kappa_{1}\right), \quad \bar{x}(0)=0, \quad \bar{v}(0)=0, \quad \overline{\tilde{x}}(0)=0, \quad \overline{\tilde{v}}(0)=0 .
$$

Then the uniform-in-time stability estimate holds, i.e., there exists a positive constant $G$ independent of $t$ such that

$$
\sup _{0 \leq t<\infty} d_{p, q}(Z(t), \tilde{Z}(t)) \leq G d_{p, q}\left(Z^{0}, \tilde{Z}^{0}\right),
$$

where the metric $d_{p, q}$ is defined as follows: for $Z=(X, V, \Theta)$ and $\tilde{Z}=(\tilde{X}, \tilde{V}, \tilde{\Theta})$,

$$
d_{p, q}(Z, \tilde{Z}):=\left(\sum_{\alpha=1}^{N}\left|x_{\alpha}-\tilde{x}_{\alpha}\right|_{p}^{q}\right)^{\frac{1}{q}}+\left(\sum_{\alpha=1}^{N}\left|v_{\alpha}-\tilde{v}_{\alpha}\right|_{p}^{q}\right)^{\frac{1}{q}}+\left(\sum_{\alpha=1}^{N}\left|\theta_{\alpha}-\tilde{\theta}_{\alpha}\right|^{q}\right)^{\frac{1}{q}} .
$$

The derivation of the uniform stability estimate (2.4) is based on the $\ell^{p}$-estimates of the variables $(X, V, \Theta)$ and the Grönwall type inequalities. Compared to the model in [14], we have only additional external potential term $x_{\alpha}$ on the righthand side of $(2.1 \mathrm{~b})$. Following the proofs of [14, Lemmas 5.2,5.3], we get additional term $|X-\tilde{X}|_{p}$, which decays to zero exponentially fast. So we can derive the Grönwall type inequalities and get the uniform stability estimate (2.4). 
Step C. (Uniform mean-field limit). We use estimates (2.3) and (2.4) to derive the global existence and asymptotic behavior of measure-valued solutions to (2.2). First, we recall Wasserstein metric $W_{p, q}(\mu, v)$ in the following definition.

Definition 2.1. For $p \in[1, \infty], q \in[1, \infty)$, let $\mathcal{P}_{q}\left(\mathbb{R}^{2 d}\right)$ be a collection of all probability measures with finite $q$-th moment: for some $z_{0} \in \mathbb{R}^{2 d}$

$$
\left\langle\mu,\left|z-z_{0}\right|_{p}^{q}\right\rangle<+\infty \text {. }
$$

Then, Wasserstein $(p, q)$-metric $W_{p, q}(\mu, v)$ is defined for any $\mu, v \in \mathcal{P}_{q}\left(\mathbb{R}^{2 d}\right)$ as

$$
W_{p, q}^{q}(\mu, v):=\inf _{\gamma \in \Gamma(\mu, v)} \int_{\mathbb{R}^{2 d} \times \mathbb{R}^{2 d}}\left|z-z^{*}\right|_{p}^{q} d \gamma\left(z, z^{*}\right), \quad W_{q}(\mu, v):=W_{q, q}(\mu, v),
$$

where $\Gamma(\mu, v)$ denotes the collection of all probability measures on $\mathbb{R}^{2 d} \times \mathbb{R}^{2 d}$ with marginals $\mu$ and $\nu$.

Suppose the initial probability measure $\mu_{0} \in \mathcal{P}\left(\mathbb{R}^{2 d} \times \mathbb{R}_{+}\right)$has a compact support in all variables and zero mean in spatial and velocity variables. Then, the following assertions hold for $p, q \in[1, \infty]$ :

1. There exists a unique measure-valued solution $\mu \in L^{\infty}\left([0, \infty) ; \mathcal{P}\left(\mathbb{R}^{2 d} \times \mathbb{R}_{+}\right)\right)$ to (2.2) such that $\mu_{t} \in \mathcal{P}\left(\mathbb{R}^{2 d} \times \mathbb{R}_{+}\right)$can be approximated by empirical measure $\mu_{t}^{N}$ in Wasserstein- $(q, p)$ distance uniformly in time

$$
\varlimsup_{N \rightarrow+\infty} \sup _{t \in[0,+\infty)} W_{q, p}\left(\mu_{t}^{n}, \mu_{t}\right)=0 .
$$

2. Moreover, if $v \in L^{\infty}\left([0, \infty) ; \mathcal{P}\left(\mathbb{R}^{2 d} \times \mathbb{R}_{+}\right)\right)$is another measure-valued solution to (2.2) with the initial datum $v_{0}$ with compact support, zero mean velocity and finite moments and the same initial condition on support with $\mu_{0}$, then there exists a nonnegative constant $\bar{G}$ independent of $t$ such that

$$
\sup _{0 \leq t<\infty} W_{q, p}\left(\mu_{t}, v_{t}\right) \leq \bar{G} W_{q, p}\left(\mu_{0}, v_{0}\right) \text {. }
$$

Similar to [14], as a direct corollary of two assertions (2.5) and (2.6), one can also obtain the flocking estimate of the measure-valued solution whose existence is guaranteed by Step A

$$
\begin{aligned}
& \left(\int_{\Omega}\|x\|_{q}^{p} d \mu_{t}\right)^{\frac{1}{p}}+\left(\int_{\Omega}\|v\|_{q}^{p} d \mu_{t}\right)^{\frac{1}{p}} \\
\leq & C e^{-\frac{1}{12} \kappa_{1} v t}\left[\left(\int_{\Omega}\|x\|_{q}^{p} d \mu_{0}\right)^{\frac{1}{p}}+\left(\int_{\Omega}\|v\|_{q}^{p} d \mu_{0}\right)^{\frac{1}{p}}\right],
\end{aligned}
$$

where $C$ is a positive constant independent of $t$. 


\subsection{The kinetic TCS model}

In this subsection, we study several properties of (1.3).

Lemma 2.2. Let $f$ be a global classical solution to system (1.3) which decays to zero sufficiently fast at infinity. Then, the following assertions hold:

1. The total mass of $f$ is conserved

$$
\int_{\Omega} f(t, z) d z=\int_{\Omega} f^{0}(z) d z, \quad t \geq 0 .
$$

2. The total energy is conserved

$$
\frac{d}{d t}\left(\int_{\Omega} \theta f d z+\frac{1}{2} \int_{\Omega}|v|^{2} f d z\right)=0, \quad t>0 .
$$

3. The averages $x_{c}$ and $v_{c}$ defined by (1.4) are governed by the harmonic oscillator equations

$$
\left\{\begin{array}{l}
\dot{x}_{c}=v_{c}, \quad t>0, \\
\dot{v}_{c}=-x_{c} .
\end{array}\right.
$$

Proof. The first and third assertions (2.7) and (2.9) can be verified easily. So we focus on the second assertion (2.8) on the energy conservation. First, we multiply $\theta$ to $(1.3 \mathrm{a})$ and integrate the resulting relation over $\Omega$ to find

$$
\begin{aligned}
\frac{d}{d t} \int_{\Omega} \theta f d z= & \kappa_{1} \int_{\Omega^{2}} \psi\left(\left|x-x_{*}\right|\right)\left(v-v_{\mathcal{C}}\right) \cdot\left(\frac{v-v_{C}}{\theta}-\frac{v_{*}-v_{C}}{\theta_{*}}\right) f f_{*} d z_{*} d z \\
& +\kappa_{2} \underbrace{\int_{\Omega^{2}} \zeta\left(\left|x-x_{*}\right|\right)\left(\frac{1}{\theta}-\frac{1}{\theta_{*}}\right) f f_{*} d z_{*} d z}_{=: \Delta}+\int_{\Omega} x \cdot v f d z \\
= & \kappa_{1} \int_{\Omega^{2}} \psi\left(\left|x-x_{*}\right|\right)\left(v-v_{C}\right) \cdot\left(\frac{v-v_{C}}{\theta}-\frac{v_{*}-v_{\mathcal{C}}}{\theta_{*}}\right) f f_{*} d z_{*} d z \quad+\int_{\Omega} x \cdot v f d z
\end{aligned}
$$

where we used the fact that

$$
\Delta=0 \quad \text { by the interchange transformation } \quad z \leftrightarrow z_{*} .
$$

Again, we use the fact that

$$
\int_{\Omega^{2}} \psi\left(\left|x-x_{*}\right|\right)\left(\frac{v-v_{\mathcal{C}}}{\theta}-\frac{v_{*}-v_{\mathcal{C}}}{\theta_{*}}\right) f f_{*} d z_{*} d z=0
$$


to see

$$
\begin{aligned}
& \frac{1}{2} \frac{d}{d t} \int_{\Omega}|v|^{2} f d z=\int_{\Omega} v \cdot \mathcal{F}[f] f d z \\
= & -\kappa_{1} \int_{\Omega^{2}} \psi\left(\left|x-x_{*}\right|\right) v \cdot\left(\frac{v-v_{c}}{\theta}-\frac{v_{*}-v_{C}}{\theta_{*}}\right) f f_{*} d z_{*} d z-\int_{\Omega} x \cdot v f d z \\
= & -\kappa_{1} \int_{\Omega^{2}} \psi\left(\left|x-x_{*}\right|\right)\left(v-v_{c}\right) \cdot\left(\frac{v-v_{c}}{\theta}-\frac{v_{*}-v_{C}}{\theta_{*}}\right) f f_{*} d z_{*} d z-\int_{\Omega} x \cdot v f d z .
\end{aligned}
$$

Finally, we combine (2.10) and (2.11) to obtain the desired estimate.

Remark 2.2. In the sequel, we comment on several ramifications by Lemma 2.2:

1. If we assume that initial total mass $\left\|f^{0}\right\|_{L^{1}}=1$, then, one has

$$
\|f(t, \cdot)\|_{L^{1}}=1, \quad t \geq 0 .
$$

2. The averages $\left(x_{c}, v_{c}\right)$ in (1.4) can be given by the following explicit formulas:

$$
x_{c}(t)=x_{c}(0) \cos t+v_{c}(0) \sin t, \quad v_{c}(t)=v_{c}(0) \cos t-x_{c}(0) \sin t .
$$

These clearly imply the uniform boundedness of the averages

$$
\left|x_{c}(t)\right|^{2} \leq 2\left(\left|v_{c}(0)\right|^{2}+\left|x_{c}(0)\right|^{2}\right), \quad\left|v_{c}(t)\right|^{2} \leq 2\left(\left|v_{c}(0)\right|^{2}+\left|x_{c}(0)\right|^{2}\right) .
$$

3. Since

$$
\int_{\Omega}|v|^{2} f d z=\int_{\Omega}\left|v-v_{c}+v_{c}\right|^{2} f d z=\int_{\Omega}\left|v-v_{c}\right|^{2} f d z+\left|v_{c}\right|^{2}
$$

the energy conservation law (2.8) can be written as

$$
\frac{d}{d t}\left(\theta_{c}+\frac{1}{2} \int_{\Omega}\left|v-v_{c}\right|^{2} f d z+\frac{1}{2}\left|v_{c}\right|^{2}\right)=0
$$

This means that

$$
\begin{aligned}
\theta_{\mathcal{C}}(t)= & \theta_{\mathcal{c}}(0)+\frac{1}{2}\left|v_{\mathcal{C}}(0)\right|^{2}+\frac{1}{2} \int_{\Omega}\left|v-v_{\mathcal{C}}(0)\right|^{2} f^{0} d z \\
& -\frac{1}{2}\left|v_{\mathcal{C}}(t)\right|^{2}-\frac{1}{2} \int_{\Omega}\left|v-v_{\mathcal{C}}\right|^{2} f d z .
\end{aligned}
$$

Before we close this section, we present a Grönwall's type lemma to be used later. 
Lemma 2.3 ([7]). Let $y:(0, \infty) \rightarrow \mathbb{R}$ be a nonnegative Lipschitz function satisfying

$$
\left\{\begin{array}{l}
y^{\prime} \leq-c_{1} y+c_{2} e^{-c_{3} t}, \quad \text { a.e. } \quad t>0 \\
y(0)=y^{0}
\end{array}\right.
$$

where $c_{1}, c_{2}$ and $c_{3}$ are positive constants. Then, one has

$$
y(t) \leq y^{0} e^{-c_{1} t}+\frac{c_{2}}{c_{1}-c_{3}}\left(e^{-c_{3} t}-e^{-c_{1} t}\right), \quad t \geq 0 .
$$

\section{Description of framework and main result}

In this section, we list our sufficient framework for emergent dynamics of (1.3) and state the main result. For this, we first recall a concept on the emergence of periodically rotating one-point cluster as follows.

Definition 3.1. Let $f=f(t, z)$ be a solution to (1.3). Then the kinetic model exhibits the emergence of periodically rotating one-point cluster asymptotically, if the following conditions hold:

1. The averaged state $\left(x_{c}, v_{c}, \theta_{c}\right)$ defined by (1.4) for thermo-mechanical observables is periodic.

2. The time-dependent supports in $z=(x, v, \theta)$ shrink asymptotically to the averaged state $\left(x_{c}, v_{c}, \theta_{c}\right)$

$$
\begin{aligned}
& \lim _{t \rightarrow \infty}\left(\left|x-x_{c}(t)\right|+\left|v-v_{c}(t)\right|+\left|\theta-\theta_{c}(t)\right|\right)=0, \\
& \forall(x, v, \theta) \in \Omega_{x}(f, t) \times \Omega_{v}(f, t) \times \Omega_{\theta}(f, t) .
\end{aligned}
$$

In what follows, we list our sufficient framework on the emergence of periodically rotating one-point cluster and provide our main result whose proof will be devoted to the following three sections.

\subsection{The framework}

For positive constants $\varepsilon_{0}>0$ and $R>0$, initial kinetic density $f^{0}$ with unit mass, we first introduce several notations

$$
\left(x_{c}^{0}, v_{c}^{0}, \theta_{c}^{0}\right):=\left(x_{c}(0), v_{c}(0), \theta_{c}(0)\right), \quad\left|\omega_{c}^{0}\right|:=\sqrt{\left|x_{c}^{0}\right|^{2}+\left|v_{c}^{0}\right|^{2}},
$$




$$
\begin{array}{ll}
\mathcal{V}_{\omega}[f]:=\int_{\Omega}\left(\left|x-x_{c}\right|^{2}+\left|v-v_{\mathcal{c}}\right|^{2}\right) f d z, & \mathcal{V}_{\theta}[f]:=\int_{\Omega}\left|\theta-\theta_{c}\right|^{2} f d z, \\
\mathcal{V}_{\omega}^{0}:=\mathcal{V}_{\omega}\left[f^{0}\right], & \mathcal{V}_{\theta}^{0}:=\mathcal{V}_{\theta}\left[f^{0}\right], \\
M:=\frac{48}{\zeta(R)} \frac{\left(\theta_{c}^{0}+2 \varepsilon_{0}\right)^{2}}{\left(\theta_{c}^{0}-2 \varepsilon_{0}\right)^{2}}, & K:=\frac{192}{\psi(R)} \frac{\left(\theta_{c}^{0}+2 \varepsilon_{0}\right)}{\left(\theta_{c}^{0}-2 \varepsilon_{0}\right)}
\end{array}
$$

Now we state the sufficient framework $(\mathcal{F})$ as follows:

$\left(\mathcal{F}_{A}\right)$ The diameter function in initial temperature and $\theta_{c}^{0}$ satisfy

$$
d_{\theta}\left[f^{0}\right]<\frac{\varepsilon_{0}}{2 \sqrt{M}}, \quad \theta_{c}^{0} \gg \varepsilon_{0} .
$$

Then it is easy to see that the initial temperature support is highly concentrated around $\theta_{c}^{0}$ and away from zero

$$
\Omega_{\theta}(f, 0) \subseteq\left(\theta_{c}^{0}-\frac{\varepsilon_{0}}{2 \sqrt{M}}, \quad \theta_{c}^{0}+\frac{\varepsilon_{0}}{2 \sqrt{M}}\right) .
$$

Based on the assumption of the initial temperature support, we make an ansatz for temperature variable: there exists $\tau>0$ such that

$$
\theta_{c}^{0}-\varepsilon_{0}<\theta_{c}(t)<\theta_{c}^{0}+\varepsilon_{0}, \quad \cup_{0 \leq t \leq \tau} \Omega_{\theta}(f, t) \subseteq B_{\varepsilon_{0}}\left(\theta_{c}(t)\right), \quad \forall t \in[0, \tau] .
$$

Then, under the above ansatz (3.3), we have

$$
\cup_{0 \leq t \leq \tau} \Omega_{\theta}(f, t) \subseteq\left(\theta_{c}^{0}-2 \varepsilon_{0}, \theta_{c}^{0}+2 \varepsilon_{0}\right) .
$$

$\left(\mathcal{F}_{B}\right)$ The diameter functions in initial position and velocity satisfy

$$
d_{x}\left[f^{0}\right]<\frac{R}{4 \sqrt{K}}, \quad d_{v}\left[f^{0}\right]<\frac{R}{4 \sqrt{K}}
$$

Then, it is easy to see

$$
\left|x-x_{c}^{0}\right|<\frac{R}{4 \sqrt{K}}, \quad\left|v-v_{c}^{0}\right|<\frac{R}{4 \sqrt{K}}, \quad \forall x \in \Omega_{x}(f, 0), \quad \forall v \in \Omega_{v}(f, 0) .
$$

Based on (3.4), we make an ansatz for mechanical variables: there exists $\tau^{*}(\leq \tau)$ such that

$$
\begin{aligned}
& \cup_{0 \leq t \leq t^{*}} \Omega_{x}(f, t) \subseteq B_{\frac{R}{2}}\left(x_{c}(t)\right) ， \\
& \cup_{0 \leq t \leq t^{*}} \Omega_{v}(f, t) \subseteq B_{\frac{R}{2}}\left(v_{c}(t)\right), \quad \forall t \in\left[0, \tau^{*}\right] .
\end{aligned}
$$


Then we use

$$
\left|x-x_{*}\right|=\left|\left(x-x_{c}\right)-\left(x_{*}-x_{c}\right)\right| \leq\left|x-x_{c}\right|+\left|x_{*}-x_{c}\right|
$$

and the ansatz (3.5) to get the uniform bounds of position- and velocity-diameter functions

$$
d_{x}[f](t)<R, \quad d_{v}[f](t)<R, \quad \forall t \in\left[0, \tau^{*}\right] .
$$

Later, we will show that $\tau^{*}=\tau$ (see the proof of Proposition 4.2).

$\left(\mathcal{F}_{C}\right) C^{2}$-communication weight functions, coupling strengths and initial configuration satisfy

$$
\begin{aligned}
& \text { (1): } \kappa_{1}>0, \kappa_{2}>0, \frac{2}{3} \frac{\kappa_{1}}{\theta_{c}^{0}-2 \varepsilon_{0}}<\frac{\kappa_{1} \psi(R)}{\theta_{c}^{0}+2 \varepsilon_{0}} \leq \frac{\kappa_{1}}{\theta_{c}^{0}-2 \varepsilon_{0}}<\frac{1}{2}, \\
& \text { (2): } \frac{\kappa_{2} \zeta(R)}{\left(\theta_{c}^{0}+2 \varepsilon_{0}\right)^{2}}>\frac{R \kappa_{1}}{\theta_{c}^{0}-2 \varepsilon_{0}}+\left(\frac{R}{2}+2 \sqrt{2}\left|\omega_{c}^{0}\right|\right), \\
& \text { (3): } \frac{\kappa_{2} \zeta(R)}{\left(\theta_{c}^{0}+2 \varepsilon_{0}\right)^{2}} \geq \frac{\kappa_{1}}{3\left(\theta_{c}^{0}-2 \varepsilon_{0}\right)} \geq \frac{\kappa_{1} \psi(R)}{3\left(\theta_{c}^{0}+2 \varepsilon_{0}\right)}, \\
& \text { (4): } 24 \frac{\theta_{c}^{0}+2 \varepsilon_{0}}{\kappa_{1} \psi(R)}\left(\frac{R \kappa_{1}}{\theta_{c}^{0}-2 \varepsilon_{0}}+\frac{R}{2}+\sqrt{2}\left|\omega_{c}^{0}\right|\right) \mathcal{V}_{\omega}^{0} \leq \frac{\varepsilon_{0}^{2}}{4 M^{\prime}}, \\
& \text { (5): } \frac{4}{3} \frac{\zeta(R)}{\left(\theta_{c}^{0}+2 \varepsilon_{0}\right)^{2}}>\frac{1}{\left(\theta_{c}^{0}-2 \varepsilon_{0}\right)^{2}}, \\
& \text { (6): } \frac{\kappa_{2} \zeta(R)}{6\left(\theta_{c}^{0}+2 \varepsilon_{0}\right)^{2}}>\frac{2 \kappa_{1}}{\theta_{c}^{0}-2 \varepsilon_{0}}+\left(\sqrt{2}\left|\omega_{c}^{0}\right|+\frac{R}{2}\right), \\
& \text { (7): } \frac{\kappa_{1}}{2\left(\theta_{c}^{0}-2 \varepsilon_{0}\right)}\left(\frac{3 R^{4}}{16}+16\left|\mathcal{V}_{\omega}^{0}\right|^{2}\right)+\frac{32\left|\mathcal{V}_{\omega}^{0}\right|^{2} \kappa_{1}}{\theta_{c}^{0}-2 \varepsilon_{0}}+\left(\sqrt{2}\left|\omega_{c}^{0}\right|+\frac{R}{2}\right) \frac{R^{2}}{4} \\
& \quad+2 R \mathcal{V}_{\omega}^{0}<\frac{\kappa_{2}}{\left(\theta_{c}^{0}-2 \varepsilon_{0}\right)^{2}} \frac{\varepsilon_{0}^{2}}{2 M^{\prime}}, \\
& \text { (8): }\left|\omega_{c}^{0}\right|^{2}+\frac{R^{2}}{8}<\varepsilon_{0}, \frac{1}{2}\left|v_{c}^{0}\right|^{2}+\frac{R^{2}}{32 K}<\varepsilon_{0} .
\end{aligned}
$$

In the following remark, we provide several comments for the above framework.

Remark 3.1. 1. If we choose

$$
\kappa_{2} \gg \kappa_{1}, \quad \theta_{c}^{0} \gg \varepsilon_{0} \gg R, \quad x_{c}^{0}=\mathcal{O}(R), \quad v_{c}^{0}=\mathcal{O}(R),
$$

and we assume that $\psi(0)=1, \psi(R), \zeta(0)=1$ and $\zeta(R)$ are comparable, then all the inequalities in (3.6) can be easily satisfied. 
2. For constant communication functions

$$
\psi(r) \equiv 1, \quad \zeta(r) \equiv 1, \quad r \geq 0,
$$

one can easily see that the conditions (1), (3) and (5) in $\left(\mathcal{F}_{C}\right)$ are always true by the second assumption in (3.2). Note that condition (6) implies (2) because we can choose $R \leq 12$. Conditions (4), (7) and (8) can be treated by the condition

$$
\left|x_{c}^{0}\right| \ll \varepsilon_{0} \quad \text { and } \quad\left|v_{c}^{0}\right| \ll \varepsilon_{0} .
$$

3. Roughly speaking, the framework $\left(\mathcal{F}_{A}\right)-\left(\mathcal{F}_{C}\right)$ can be stated as follows:

- The initial temperature support is highly concentrated around $\theta_{c}^{0}$ and is far away from zero.

- The initial position, initial velocity and their initial diameters are much smaller than the initial temperature-diameter.

\subsection{Main result}

In this subsection, we briefly state our main result and its corollary.

Theorem 3.1. Suppose the framework $\left(\mathcal{F}_{A}\right)-\left(\mathcal{F}_{C}\right)$ in Section 3.1 holds, and let $f$ be a global classical solution to (1.3). One has the following two assertions:

1. (Exponential decay of variances). The variances in (3.1) decay exponentially fast

$$
\mathcal{V}_{\omega}[f](t) \leq 4 \mathcal{V}_{\omega}^{0} e^{-\frac{\kappa_{1} \psi(R)}{6\left(\theta_{c}^{0}+2 \varepsilon_{0}\right)} t}, \quad \mathcal{V}_{\theta}[f](t) \leq \frac{\varepsilon_{0}^{2}}{2 M} e^{-\frac{\kappa_{1} \psi(R)}{6\left(\theta_{c}^{0}+2 \varepsilon_{0}\right)} t}, \quad t \geq 0
$$

2. (Exponential emergence of one-point cluster). The diameters $d_{x}[f], d_{v}[f]$ and $d_{\theta}[f]$ decay to zero exponentially fast

$$
\begin{array}{ll}
\Omega_{\theta}(f, t) \subseteq\left(\theta_{c}^{0}-2 \varepsilon_{0}, \theta_{c}^{0}+2 \varepsilon_{0}\right), & d_{x}[f](t) \leq \operatorname{Re}^{-\frac{\kappa_{1} \psi(R)}{24\left(\theta_{c}^{0}+2 \varepsilon_{0}\right)} t}, \\
d_{v}[f](t) \leq \operatorname{Re}^{-\frac{\kappa_{1} \psi(R)}{24\left(\theta_{c}^{0}+2 \varepsilon_{0}\right)} t}, & d_{\theta}[f](t) \leq \varepsilon_{0} e^{-\frac{\kappa_{1} \psi(R)}{24\left(\theta_{c}^{0}+2 \varepsilon_{0}\right)} t} .
\end{array}
$$

Proof. The following three sections are devoted to the proof of this theorem. 
The variance estimates in (3.7) are also true for the measure-valued solution to (1.3) if we modify our proof using a similar argument as in [6,17]. This implies that $f$ converges to the multiple of Dirac measure concentrated on the averaged state $\left(x_{c}(t), v_{\mathcal{c}}(t), \theta_{c}(t)\right)$ in the generalized phase space $(x, v, \theta)$ as $t \rightarrow \infty$. To quantify this, we will measure the distance between the one-particle kinetic density $f$ and the multiple of Dirac measure in "the bounded Lipschitz distance": Let $\mu$ and $v$ be two measures

$$
\begin{aligned}
& \operatorname{dist}_{B L}(\mu, v) \\
:= & \sup \left\{\int_{\Omega} \phi(z) d \mu(z)-\int_{\Omega} \phi(z) d v(z):\|\phi\|_{L^{\infty}} \leq 1,\|\phi\|_{L i p}:=\sup _{z \neq z_{*}} \frac{\left|\phi(z)-\phi\left(z_{*}\right)\right|}{\left|z-z_{*}\right|} \leq 1\right\} .
\end{aligned}
$$

Corollary 3.1. Suppose the framework $\left(\mathcal{F}_{A}\right)-\left(\mathcal{F}_{C}\right)$ in Section 3.1 holds, and let $f$ be a global classical solution to (1.3). Then $f$ tends to the Dirac measure $\delta_{z_{c}(t)}:=\delta_{x_{c}} \otimes \delta_{v_{c}} \otimes \delta_{\theta_{c}}$ centered at $z_{c}$ asymptotically

$$
\lim _{t \rightarrow+\infty} \operatorname{dist}_{B L}\left(f, \delta_{z_{\mathcal{c}}(t)}\right)=0 .
$$

Proof. We use the same argument as in [8]. For any bounded Lipschitz functions $\phi=\phi(z)$ with $\|\phi\|_{\text {Lip }} \leq 1$, we get

$$
\begin{aligned}
& \left|\int_{\Omega} \phi(z) f(t, z) d z-\int_{\Omega} \phi(z) \delta_{z_{c}(t)} d z\right| \\
= & \left|\int_{\Omega}\left(\phi(z)-\phi\left(z_{c}(t)\right)\right) f(t, z) d z\right| \\
\leq & \|\phi\|_{L i p} \int_{\Omega}\left(\left|x-x_{c}\right|+\left|v-v_{\mathcal{C}}\right|+\left|\theta-\theta_{\mathcal{C}}\right|\right) f(t, z) d z \\
\leq & \int_{\Omega}\left(\left|x-x_{\mathcal{c}}\right|^{2}+\left|v-v_{\mathcal{C}}\right|^{2}+\left|\theta-\theta_{\mathcal{C}}\right|^{2}\right) f(t, z) d z \\
= & \mathcal{V}_{\omega}[f](t)+\mathcal{V}_{\theta}[f](t),
\end{aligned}
$$

where in the last second inequality, we used the Cauchy-Schwarz inequality using the unit mass condition $\int_{\Omega} f(t, z) d z=1$. Then, we apply the variance estimates in (3.7) for (3.8) to derive the desired estimate.

In next three sections, we provide the proof of the uniform bounds of diameter functions and a periodical rotationally one-point cluster flocking results in the sense of Definition 3.1, formulated in terms of initial configuration, coupling strengths and communication weights.

Below, we briefly outline a proof strategy in three steps. Based on the assumptions of (3.2) and (3.4), we make two ansatzs (3.3) and (3.5): 
Step A. (Concentration of mechanical variables in a small-time interval). We introduce forward characteristic curves associated with the kinetic full TCS equation (1.3). Under the two ansatzs, we provide the concentration estimate of mechanical variables around the harmonic oscillator motion using the exponential decay result of variance functional measuring mechanical fluctuation, improve the second ansatz (3.5) (see Section 4).

Step B. (Concentration of temperature variable in a small-time interval). Under the two ansatzs, we provide the concentration estimate of temperature variable around the averaged value using the exponential decay result of the variance functional measuring temperature fluctuation, improve the first ansatz (3.3) (see Section 5).

Step C. (Removal of ansatzs and extension of concentrations in the whole-time interval). We remove the ansatzs (3.3) and (3.5) using the continuity arguments and prove Theorem 3.1 (see Section 6).

\section{Concentration of mechanical variables}

In this section, we provide the concentration estimate of mechanical variables around the harmonic oscillator motion via a variance functional in a small-time interval to improve the second ansatz (3.5).

\subsection{Decay estimate of variance functional in mechanical variables}

In this subsection, we study exponential decay estimate of the variance functional $\mathcal{V}_{\omega}[f]$ via an alternative equivalent functional. For this, we introduce a Lyapunov functional which is equivalent to $\mathcal{V}_{\omega}[f]$

$$
\mathcal{L}_{\omega}[f]:=\int_{\Omega}\left(\frac{1}{2}\left|x-x_{\mathcal{c}}\right|^{2}+\frac{1}{2}\left|v-v_{\mathcal{C}}\right|^{2}+\lambda\left(x-x_{\mathcal{c}}\right) \cdot\left(v-v_{\mathcal{C}}\right)\right) f d z
$$

Note that for $0<\lambda \leq 1 / 2$, by using the following inequalities:

$$
\begin{aligned}
& \frac{1}{2}\left|x-x_{c}\right|^{2}+\frac{1}{2}\left|v-v_{c}\right|^{2}+\lambda\left(x-x_{c}\right) \cdot\left(v-v_{c}\right) \\
\geq & \frac{1}{4}\left[\left|x-x_{c}+\lambda\left(v-v_{c}\right)\right|^{2}+\left|v-v_{c}+\lambda\left(x-x_{c}\right)\right|^{2}+\left(1-\lambda^{2}\right)\left(\left|x-x_{c}\right|^{2}+\left|v-v_{c}\right|^{2}\right)\right]
\end{aligned}
$$




$$
\begin{aligned}
\geq & \frac{1}{4}\left(1-\lambda^{2}\right)\left(\left|x-x_{c}\right|^{2}+\left|v-v_{\mathcal{C}}\right|^{2}\right), \\
& \frac{1}{2}\left|x-x_{c}\right|^{2}+\frac{1}{2}\left|v-v_{\mathcal{c}}\right|^{2}+\lambda\left(x-x_{c}\right) \cdot\left(v-v_{\mathcal{C}}\right) \leq \frac{1}{2}(1+\lambda)\left(\left|x-x_{c}\right|^{2}+\left|v-v_{\mathcal{C}}\right|^{2}\right),
\end{aligned}
$$

one can see that the variance functional $\mathcal{V}_{\omega}[f]$ defined by (3.1) is equivalent to $\mathcal{L}_{\omega}[f]$

$$
\frac{3}{16} \mathcal{V}_{\omega}[f] \leq \mathcal{L}_{\omega}[f] \leq \frac{3}{4} \mathcal{V}_{\omega}[f]
$$

In next lemma, we provide time-evolution estimate of the components of $\mathcal{L}_{\omega}[f]$ as follows.

Lemma 4.1. Suppose the framework $\left(\mathcal{F}_{A}\right)-\left(\mathcal{F}_{C}\right)$ holds, and let $f$ be the global classical solution to (1.3). Then, the component functionals of $\mathcal{L}_{\omega}[f]$ satisfy the following estimates:

(i) $\frac{d}{d t} \int_{\Omega}\left|x-x_{c}\right|^{2} f d z=2 \int_{\Omega}\left(x-x_{c}\right) \cdot\left(v-v_{c}\right) f d z$;

(ii) $\frac{d}{d t} \int_{\Omega}\left|v-v_{\mathcal{C}}\right|^{2} f d z$

$$
\leq-2 \kappa_{1}\left(\frac{\psi\left(d_{x}[f]\right)}{\theta_{c}^{0}+2 \varepsilon_{0}}-\frac{2 \varepsilon_{0}}{\left(\theta_{c}^{0}-2 \varepsilon_{0}\right)^{2}}\right) \int_{\Omega}\left|v-v_{\mathcal{c}}\right|^{2} f d z-2 \int_{\Omega}\left(v-v_{\mathcal{C}}\right) \cdot\left(x-x_{c}\right) f d z ;
$$

(iii) $\frac{d}{d t} \int_{\Omega}\left(x-x_{c}\right) \cdot\left(v-v_{c}\right) f d z$

$$
\leq-\frac{1}{2} \int_{\Omega}\left|x-x_{c}\right|^{2} f d z+\left(1+\frac{2\left(\kappa_{1}\right)^{2}}{\left(\theta_{c}^{0}-2 \varepsilon_{0}\right)^{2}}\right) \int_{\Omega}\left|v-v_{c}\right|^{2} f d z \text {. }
$$

Proof. Suppose that the assumptions (3.2), (3.4) and (3.6) hold, and we make two ansatzs (3.3) and (3.5) on the supports of $f$ in $z=(x, v, \theta)$ in a small-time interval and we get

$$
\cup_{0 \leq t \leq \tau} \Omega_{\theta}(f, t) \subseteq\left(\theta_{c}^{0}-2 \varepsilon_{0}, \quad \theta_{c}^{0}+2 \varepsilon_{0}\right), \quad d_{x}[f](t) \leq R, \quad \forall t \in\left[0, \tau^{*}\right] \subseteq[0, \tau] .
$$

(i) By definition of $x_{c}$, one has

$$
\int_{\Omega}\left(x-x_{c}\right) f d z=0
$$

Then, we use the above relation and $(1.3)_{1}$ to find

$$
\frac{d}{d t} \int_{\Omega}\left|x-x_{c}\right|^{2} f d z=-2 \frac{d x_{c}}{d t} \cdot \int_{\Omega}\left(x-x_{c}\right) f d z+\int_{\Omega}\left|x-x_{c}\right|^{2} \partial_{t} f d z
$$




$$
\begin{aligned}
& =-\int_{\Omega}\left|x-x_{c}\right|^{2}\left[\nabla_{x} \cdot(v f)+\nabla_{v} \cdot(\mathcal{F}[f] f)+\partial_{\theta}(\mathcal{G}[f] f)\right] d z \\
& =2 \int_{\Omega}\left(x-x_{c}\right) \cdot v f d z=2 \int_{\Omega}\left(x-x_{c}\right) \cdot\left(v-v_{c}+v_{c}\right) f d z \\
& =2 \int_{\Omega}\left(x-x_{c}\right) \cdot\left(v-v_{c}\right) f d z .
\end{aligned}
$$

(ii) Note that

$$
\frac{v-v_{c}}{\theta}-\frac{v_{*}-v_{c}}{\theta_{*}}=\frac{v-v_{*}}{\theta}+\left(v_{*}-v_{c}\right)\left(\frac{1}{\theta}-\frac{1}{\theta_{*}}\right)
$$

Now, we use (1.3a), (1.3b), the relations

$$
\int_{\Omega}\left(v-v_{\mathcal{C}}\right) f d z=0, \quad \psi\left(\left|x-x_{*}\right|\right)=\psi\left(\left|x_{*}-x\right|\right)
$$

and interchange transformation $v \leftrightarrow v_{*}$ to obtain

$$
\begin{aligned}
& \frac{d}{d t} \int_{\Omega}\left|v-v_{\mathcal{C}}\right|^{2} f d z=-2 \frac{d v_{\mathcal{C}}}{d t} \cdot \int_{\Omega}\left(v-v_{\mathcal{C}}\right) f d z+\int_{\Omega}\left|v-v_{\mathcal{C}}\right|^{2} \partial_{t} f d z \\
= & 2 \int_{\Omega}\left(v-v_{\mathcal{C}}\right) \cdot \mathcal{F}[f] f d z \\
= & -2 \kappa_{1} \int_{\Omega^{2}} \psi\left(\left|x-x_{*}\right|\right)\left(v-v_{\mathcal{C}}\right) \cdot\left(\frac{v-v_{\mathcal{C}}}{\theta}-\frac{v_{*}-v_{\mathcal{C}}}{\theta_{*}}\right) f_{*} f d z_{*} d z \\
& -2 \int_{\Omega}\left(v-v_{\mathcal{C}}\right) \cdot\left(x-x_{\mathcal{C}}\right) f d z \\
= & -\kappa_{1} \int_{\Omega^{2}} \psi\left(\left|x-x_{*}\right|\right)\left(v-v_{*}\right) \cdot\left(\frac{v-v_{\mathcal{C}}}{\theta}-\frac{v_{*}-v_{\mathcal{C}}}{\theta_{*}}\right) f_{*} f d z_{*} d z \\
& -2 \int_{\Omega}\left(v-v_{\mathcal{C}}\right) \cdot\left(x-x_{\mathcal{C}}\right) f d z \\
= & -\kappa_{1} \int_{\Omega^{2}} \psi\left(\left|x-x_{*}\right|\right) \frac{\left|v-v_{*}\right|^{2}}{\theta} f_{*} f d z_{*} d z \\
& -\kappa_{1} \int_{\Omega^{2}} \psi\left(\left|x-x_{*}\right|\right)\left(v_{*}-v_{\mathcal{C}}\right) \cdot\left(v-v_{*}\right)\left(\frac{1}{\theta}-\frac{1}{\theta_{*}}\right) f_{*} f d z_{*} d z \\
& -2 \int_{\Omega}\left(v-v_{\mathcal{C}}\right) \cdot\left(x-x_{c}\right) f d z \\
= & : \mathcal{I}_{11}+\mathcal{I}_{12}+\mathcal{I}_{13} \cdot
\end{aligned}
$$

In what follows, we provide estimates for $\mathcal{I}_{1 i}$ one by one. 
- (Estimate for $\left.\mathcal{I}_{11}\right)$. We use

$$
\int_{\Omega}\left(v-v_{c}\right) f d z=0
$$

to see

$$
\begin{aligned}
\mathcal{I}_{11} & \leq-\frac{\kappa_{1} \psi\left(d_{x}[f]\right)}{\left(\theta_{c}^{0}+2 \varepsilon_{0}\right)} \int_{\Omega^{2}}\left|v-v_{*}\right|^{2} f_{*} f d z_{*} d z \\
& =-\frac{2 \kappa_{1} \psi\left(d_{x}[f]\right)}{\theta_{c}^{0}+2 \varepsilon_{0}} \int_{\Omega}\left|v-v_{\mathcal{c}}\right|^{2} f d z .
\end{aligned}
$$

- (Estimate for $\left.\mathcal{I}_{12}\right)$. We use

$$
\psi(r) \leq \psi(0)=1, \quad\left|\frac{1}{\theta}-\frac{1}{\theta_{*}}\right|=\frac{\left|\theta-\theta_{*}\right|}{|\theta| \cdot\left|\theta_{*}\right|} \leq \frac{\left|\theta-\theta_{*}\right|}{\theta_{m}^{2}}, \quad d_{\theta}[f] \leq 4 \varepsilon_{0}
$$

to get

$$
\begin{aligned}
\left|\mathcal{I}_{12}\right| & =\left|-\frac{\kappa_{1}}{2} \int_{\Omega^{2}} \psi\left(\left|x-x_{*}\right|\right)\left(\left|v-v_{\mathcal{c}}\right|^{2}-\left|v_{*}-v_{\mathcal{c}}\right|^{2}\right)\left(\frac{1}{\theta}-\frac{1}{\theta_{*}}\right) f_{*} f d z_{*} d z\right| \\
& \leq \frac{\kappa_{1} d_{\theta}[f]}{2\left(\theta_{c}^{0}-2 \varepsilon_{0}\right)^{2}} \int_{\Omega^{2}}\left(\left|v-v_{\mathcal{C}}\right|^{2}+\left|v_{*}-v_{\mathcal{C}}\right|^{2}\right) f_{*} f d z_{*} d z \\
& \leq \frac{4 \kappa_{1} \varepsilon_{0}}{\left(\theta_{c}^{0}-2 \varepsilon_{0}\right)^{2}} \int_{\Omega}\left|v-v_{\mathcal{C}}\right|^{2} f d z .
\end{aligned}
$$

In (4.4), we combine (4.5) and (4.6) to find

$$
\begin{aligned}
\frac{d}{d t} \int_{\Omega}\left|v-v_{\mathcal{C}}\right|^{2} f d z \leq & -2 \kappa_{1}\left(\frac{\psi\left(d_{x}[f]\right)}{\theta_{c}^{0}+2 \varepsilon_{0}}-\frac{2 \varepsilon_{0}}{\left(\theta_{c}^{0}-2 \varepsilon_{0}\right)^{2}}\right) \int_{\Omega}\left|v-v_{\mathcal{C}}\right|^{2} f d z \\
& -2 \int_{\Omega}\left(v-v_{\mathcal{C}}\right) \cdot\left(x-x_{c}\right) f d z .
\end{aligned}
$$

(iii) By straightforward calculation, one has

$$
\begin{aligned}
& \frac{d}{d t} \int_{\Omega}\left(x-x_{\mathcal{c}}\right) \cdot\left(v-v_{\mathcal{C}}\right) f d z=\int_{\Omega}\left(x-x_{\mathcal{C}}\right) \cdot\left(v-v_{\mathcal{C}}\right) \partial_{t} f d z \\
= & -\int_{\Omega}\left(x-x_{\mathcal{c}}\right) \cdot\left(v-v_{\mathcal{C}}\right) \nabla_{x} \cdot(v f) d z-\int_{\Omega}\left(x-x_{\mathcal{c}}\right) \cdot\left(v-v_{\mathcal{C}}\right) \nabla_{v} \cdot(\mathcal{F}[f] f) d z \\
= & \int_{\Omega}\left|v-v_{\mathcal{C}}\right|^{2} f d z+\int_{\Omega}\left(x-x_{\mathcal{c}}\right) \cdot \mathcal{F}[f] f d z \\
= & \int_{\Omega}\left|v-v_{\mathcal{C}}\right|^{2} f d z-\int_{\Omega}\left|x-x_{\mathcal{c}}\right|^{2} f d z
\end{aligned}
$$




$$
\begin{aligned}
& -\kappa_{1} \int_{\Omega^{2}} \psi\left(\left|x-x_{*}\right|\right)\left(x-x_{c}\right) \cdot\left(\frac{v-v_{c}}{\theta}-\frac{v_{*}-v_{c}}{\theta_{*}}\right) f_{*} f d z_{*} d z \\
\leq & -\frac{1}{2} \int_{\Omega}\left|x-x_{\mathcal{c}}\right|^{2} f d z+\left(1+\frac{2\left(\kappa_{1}\right)^{2}}{\left(\theta_{c}^{0}-2 \varepsilon_{0}\right)^{2}}\right) \int_{\Omega}\left|v-v_{\mathcal{C}}\right|^{2} f d z,
\end{aligned}
$$

where we used the following estimates via Young's inequality:

$$
\begin{aligned}
& -\kappa_{1} \int_{\Omega^{2}} \psi\left(\left|x-x_{*}\right|\right)\left(x-x_{c}\right) \cdot\left(\frac{v-v_{c}}{\theta}-\frac{v_{*}-v_{C}}{\theta_{*}}\right) f_{*} f d z_{*} d z \\
\leq & \frac{\kappa_{1}}{2} \int_{\Omega^{2}}\left[\frac{1}{\kappa_{1}}\left|x-x_{c}\right|^{2}+2 \kappa_{1}\left(\frac{\left|v-v_{\mathcal{C}}\right|^{2}}{\theta^{2}}+\frac{\left|v_{*}-v_{\mathcal{C}}\right|^{2}}{\theta_{*}^{2}}\right)\right] f_{*} f d z_{*} d z \\
\leq & \frac{1}{2} \int_{\Omega}\left|x-x_{c}\right|^{2} f d z+\frac{2\left(\kappa_{1}\right)^{2}}{\left(\theta_{c}^{0}-2 \varepsilon_{0}\right)^{2}} \int_{\Omega}\left|v-v_{\mathcal{C}}\right|^{2} f d z .
\end{aligned}
$$

The proof is complete.

Remark 4.1. Note that from the first two estimates in Lemma 4.1, one has

$$
\frac{d}{d t} \mathcal{V}_{\omega}[f] \leq-2 \kappa_{1}\left(\frac{\left.\psi\left(d_{x}[f]\right)\right)}{\theta_{c}^{0}+2 \varepsilon_{0}}-\frac{2 \varepsilon_{0}}{\left(\theta_{c}^{0}-2 \varepsilon_{0}\right)^{2}}\right) \int_{\Omega}\left|v-v_{\mathcal{c}}\right|^{2} f d z
$$

Since the R.H.S. of the above relation does not contain the term $\int_{\Omega}\left|x-x_{c}\right|^{2} f d z$, we cannot derive the Grönwall's inequality for $\mathcal{V}_{\omega}[f]$. That is why we consider the equivalent functional $\mathcal{L}_{\omega}[f]$ containing the cross term $\int_{\Omega}\left(x-x_{c}\right) \cdot\left(v-v_{c}\right) f d z$.

Now, we are ready to provide exponential decay estimate for $\mathcal{V}_{\omega}[f]$.

Proposition 4.1. Suppose the framework $\left(\mathcal{F}_{A}\right)-\left(\mathcal{F}_{C}\right)$ holds, and let $f$ be the global classical solution to (1.3). Then, there exist some positive constants $\tau$ and $\tau^{*}$, such that for $t \in\left[0, \tau^{*}\right] \subseteq[0, \tau]$

$$
\mathcal{V}_{\omega}[f](t) \leq 4 \mathcal{V}_{\omega}\left[f^{0}\right] e^{-\frac{\kappa_{1} \psi(R)}{6\left(\theta_{c}^{0}+2 \varepsilon_{0}\right)} t}
$$

Proof. Consider the linear combination

$$
\frac{1}{2}(4.3)+\frac{1}{2}(4.7)+\lambda(4.8), \quad \lambda=\frac{\kappa_{1} \psi(R)}{4\left(\theta_{c}^{0}+2 \varepsilon_{0}\right)}<\frac{1}{2}
$$

to find

$$
\frac{d}{d t} \mathcal{L}_{\omega}[f]=-\left[\frac{\kappa_{1} \psi\left(d_{x}[f]\right)}{\theta_{c}^{0}+2 \varepsilon_{0}}-\frac{2 \kappa_{1} \varepsilon_{0}}{\left(\theta_{c}^{0}-2 \varepsilon_{0}\right)^{2}}-\lambda\left(1+\frac{2\left(\kappa_{1}\right)^{2}}{\left(\theta_{c}^{0}-2 \varepsilon_{0}\right)^{2}}\right)\right]
$$




$$
\begin{aligned}
& \times \int_{\Omega}\left|v-v_{\mathcal{c}}\right|^{2} f d z-\frac{\lambda}{2} \int_{\Omega}\left|x-x_{\mathcal{c}}\right|^{2} f d z \\
\leq- & \frac{\kappa_{1} \psi(R)}{8\left(\theta_{c}^{0}+2 \varepsilon_{0}\right)} \int_{\Omega}\left(\left|v-v_{\mathcal{c}}\right|^{2}+\left|x-x_{c}\right|^{2}\right) f d z=-\frac{\kappa_{1} \psi(R)}{8\left(\theta_{c}^{0}+2 \varepsilon_{0}\right)} \mathcal{V}_{\omega}[f] \\
\leq- & \frac{\kappa_{1} \psi(R)}{6\left(\theta_{c}^{0}+2 \varepsilon_{0}\right)} \mathcal{L}_{\omega}[f],
\end{aligned}
$$

where we used the relation (1) in (3.6)

$$
\frac{2}{3} \frac{\kappa_{1}}{\theta_{c}^{0}-2 \varepsilon_{0}}<\frac{\kappa_{1} \psi(R)}{\theta_{c}^{0}+2 \varepsilon_{0}} \leq \frac{\kappa_{1}}{\theta_{c}^{0}-2 \varepsilon_{0}}<\frac{1}{2}, \quad \varepsilon_{0} \ll \theta_{c}^{0} .
$$

Then, Lemma 2.3 yields

$$
\mathcal{L}_{\omega}[f](t) \leq e^{-\frac{\kappa_{1} \psi(R) t}{6\left(\theta_{c}^{0}+2 \varepsilon_{0}\right)}} \mathcal{L}_{\omega}\left[f^{0}\right], \quad t \geq 0
$$

Finally, we use (4.2) and (4.9) to derive desired estimate

$$
\mathcal{V}_{\omega}[f](t) \leq \frac{16}{3} \mathcal{L}_{\omega}[f](t) \leq \frac{16}{3} e^{-\frac{\kappa_{1} \psi(R) t}{6\left(\theta_{c}^{0}+2 \varepsilon_{0}\right)}} \mathcal{L}_{\omega}\left[f^{0}\right] \leq 4 e^{-\frac{\kappa_{1} \psi(R) t}{6\left(\theta_{c}^{0}+2 \varepsilon_{0}\right)}} \mathcal{V}_{\omega}\left[f^{0}\right]
$$

The proof is complete.

\subsection{Pointwise exponential concentration of mechanical variables}

Let $z=(x, v, \theta)$ be a given triple of position, velocity and temperature. Then, we define the forward characteristics passing through $z$ at time 0

$$
(x(s), v(s), \theta(s)):=(x(s ; 0, x, v, \theta), v(s ; 0, x, v, \theta), \theta(s ; 0, x, v, \theta))
$$

as a unique solution to the following Cauchy problem:

$$
\left\{\begin{array}{l}
\frac{d x(s)}{d s}=v(s), \quad s>0 \\
\frac{d v(s)}{d s}=\mathcal{F}[f](x(s), v(s), \theta(s), s), \\
\frac{d \theta(s)}{d s}=\mathcal{G}[f](x(s), \theta(s), s) \\
(x(0), v(0), \theta(0))=(x, v, \theta),
\end{array}\right.
$$


where $\mathcal{F}[f]$ and $\mathcal{G}[f]$ are nonlocal functionals introduced in (1.3)

$$
\begin{aligned}
\mathcal{F}[f](x, v, \theta):= & -\kappa_{1} \int_{\Omega} \psi\left(\left|x-x_{*}\right|\right)\left(\frac{v-v_{\mathcal{C}}}{\theta}-\frac{v_{*}-v_{\mathcal{c}}}{\theta_{*}}\right) f_{*} d z_{*}-x, \\
\mathcal{G}[f](x, v, \theta):= & \kappa_{1} \int_{\Omega} \psi\left(\left|x-x_{*}\right|\right)\left(v-v_{\mathcal{c}}\right) \cdot\left(\frac{v-v_{\mathcal{C}}}{\theta}-\frac{v_{*}-v_{c}}{\theta_{*}}\right) f_{*} d z_{*} \\
& +\kappa_{2} \int_{\Omega} \zeta\left(\left|x-x_{*}\right|\right)\left(\frac{1}{\theta}-\frac{1}{\theta_{*}}\right) f_{*} d z_{*}+x \cdot v .
\end{aligned}
$$

The Lipschitz continuous properties of the communication weight functions and the uniform boundedness estimates for $x, v$ and $\theta$ (see Propositions 4.2 and 5.2) imply that the right-hand side of (4.10) is bounded, thus the forward characteristics are globally in time well-defined. On the other hand, it follows from (2.9) and (2.10) that the averages satisfy

$$
\frac{d x_{c}(s)}{d s}=v_{\mathcal{C}}(s), \quad \frac{d v_{\mathcal{c}}(s)}{d s}=-x_{c}(s), \quad \frac{d \theta_{c}(s)}{d s}=\int_{\Omega} \mathcal{G}[f] f d z, \quad s>0 .
$$

Now, we consider the fluctuations $(\hat{x}(t), \hat{v}(t), \hat{\theta}(t))$ around the averages

$$
\hat{x}(t):=x(t)-x_{c}(t), \quad \hat{v}(t):=v(t)-v_{\mathcal{c}}(t), \quad \hat{\theta}(t):=\theta(t)-\theta_{c}(t) .
$$

Then, it follows from (4.10) and (4.11) that the fluctuations $(\hat{x}, \hat{v}, \hat{\theta})$ satisfy

$$
\left\{\begin{array}{l}
\frac{d \hat{x}(s)}{d s}=\hat{v}(s), \quad s>0, \\
\frac{d \hat{v}(s)}{d s}=-\kappa_{1} \int_{\Omega} \psi\left(\left|x(s)-x_{*}\right|\right)\left(\frac{\hat{v}(s)}{\theta(s)}-\frac{\hat{v}_{*}}{\theta_{*}}\right) f_{*} d z_{*}-\hat{x}(s), \\
\frac{d \tilde{\theta}(s)}{d s}=\mathcal{G}[f](s, x(s), \theta(s))-\int_{\Omega} \mathcal{G}[f] f d z,
\end{array}\right.
$$

where we assumed that $\int_{\Omega} f(t, z) d z=1$.

Lemma 4.2. Suppose the framework $\left(\mathcal{F}_{A}\right)-\left(\mathcal{F}_{C}\right)$ holds, and let $f$ be the global classical solution to (1.3). Then, the fluctuations $(\hat{x}, \hat{v}, \hat{\theta})$ satisfy

(i) $\frac{d}{d s}\left(\frac{1}{2}|\hat{x}|^{2}\right)=\hat{x} \cdot \hat{v}$;

(ii) $\frac{d}{d s}\left(\frac{1}{2}|\hat{v}|^{2}\right) \leq-\frac{\kappa_{1} \psi\left(d_{x}[f]\right)}{\theta_{c}^{0}+2 \varepsilon_{0}}|\hat{v}|^{2}+\frac{\kappa_{1}}{2\left(\theta_{c}^{0}-2 \varepsilon_{0}\right)}\left(|\hat{v}(s)|^{2}+\mathcal{V}_{\omega}[f]\right)-\hat{x} \cdot \hat{v}$; 
(iii) $\frac{d}{d s}(\hat{x} \cdot \hat{v}) \leq|\hat{v}|^{2}+\frac{\kappa_{1}}{2\left(\theta_{c}^{0}-2 \varepsilon_{0}\right)}\left(2|\hat{x}|^{2}+|\hat{v}|^{2}+\mathcal{V}_{\omega}[f]\right)-|\hat{x}|^{2}$.

Proof. Let $(\hat{x}(s), \hat{v}(s), \hat{\theta}(s))$ be the forward characteristics defined in (4.12). In what follows, we provide estimates for the following terms:

$$
\frac{1}{2}|\hat{x}(s)|^{2}, \quad \frac{1}{2}|\hat{v}(s)|^{2}, \quad \hat{x}(s) \cdot \hat{v}(s) .
$$

(i) We use $(4.12 a)$ to find

$$
\frac{d}{d s}\left(\frac{1}{2}|\hat{x}(s)|^{2}\right)=\hat{x}(s) \cdot \frac{d \hat{x}(s)}{d s}=\hat{x}(s) \cdot \hat{v}(s) .
$$

(ii) We use (4.12b) and Young's inequality to get

$$
\begin{aligned}
& \frac{d}{d s}\left(\frac{1}{2}|\hat{v}(s)|^{2}\right)=\hat{v}(s) \cdot \frac{d \hat{v}(s)}{d s} \\
= & -\kappa_{1} \hat{v}(s) \cdot \int_{\Omega} \psi\left(\left|x(s)-x_{*}\right|\right)\left(\frac{\hat{v}(s)}{\theta(s)}-\frac{\hat{v}_{*}}{\theta_{*}}\right) f_{*} d z_{*}-\hat{x}(s) \cdot \hat{v}(s) \\
\leq & -\frac{\kappa_{1} \psi\left(d_{x}[f]\right)}{\theta_{c}^{0}+2 \varepsilon_{0}}|\hat{v}(s)|^{2}+\kappa_{1} \int_{\Omega} \psi\left(\left|x(s)-x_{*}\right|\right) \frac{\hat{v}_{*} \cdot \hat{v}(s)}{\theta_{*}} f_{*} d z_{*}-\hat{x}(s) \cdot \hat{v}(s) \\
\leq & -\frac{\kappa_{1} \psi\left(d_{x}[f]\right)}{\theta_{c}^{0}+2 \varepsilon_{0}}|\hat{v}(s)|^{2}+\frac{\kappa_{1}}{\theta_{c}^{0}-2 \varepsilon_{0}}|\hat{v}(s)|\left(\int_{\Omega}\left|\hat{v}_{*}\right|^{2} f_{*} d z_{*}\right)^{\frac{1}{2}}-\hat{x}(s) \cdot \hat{v}(s) \\
\leq & -\frac{\kappa_{1} \psi\left(d_{x}[f]\right)}{\theta_{c}^{0}+2 \varepsilon_{0}}|\hat{v}(s)|^{2}+\frac{\kappa_{1}}{2\left(\theta_{c}^{0}-2 \varepsilon_{0}\right)}\left(|\hat{v}(s)|^{2}+\mathcal{V}_{\omega}[f](s)\right)-\hat{x}(s) \cdot \hat{v}(s) .
\end{aligned}
$$

(iii) Similarly, one has

$$
\begin{aligned}
& \frac{d}{d s}(\hat{x}(s) \cdot \hat{v}(s)) \\
= & |\hat{v}(s)|^{2}-\kappa_{1} \hat{x}(s) \cdot \int_{\Omega} \psi\left(\left|x(s)-x_{*}\right|\right)\left(\frac{\hat{v}(s)}{\theta(s)}-\frac{\hat{v}_{*}(s)}{\theta_{*}}\right) f_{*} d z_{*}-|\hat{x}(s)|^{2} \\
\leq & |\hat{v}(s)|^{2}+|\hat{x}(s)| \frac{\kappa_{1}}{\theta_{c}^{0}-2 \varepsilon_{0}}\left(|\hat{v}(s)|+\left(\int_{\Omega}\left|\hat{v}_{*}(s)\right|^{2} f_{*} d z_{*}\right)^{\frac{1}{2}}\right)-|\hat{x}(s)|^{2} \\
\leq & |\hat{v}(s)|^{2}+|\hat{x}(s)| \frac{\kappa_{1}}{\theta_{c}^{0}-2 \varepsilon_{0}}\left(|\hat{v}(s)|+\sqrt{\mathcal{V}_{\omega}[f](s)}\right)-|\hat{x}(s)|^{2} \\
\leq & |\hat{v}(s)|^{2}+\frac{\kappa_{1}}{2\left(\theta_{c}^{0}-2 \varepsilon_{0}\right)}\left(2|\hat{x}(s)|^{2}+|\hat{v}(s)|^{2}+\mathcal{V}_{\omega}[f](s)\right)-|\hat{x}(s)|^{2} .
\end{aligned}
$$

The proof is complete. 
Now we state the pointwise decay estimate result of mechanical fluctuation around the harmonic oscillator motion along forward characteristics.

Proposition 4.2. Suppose the framework $\left(\mathcal{F}_{A}\right)-\left(\mathcal{F}_{C}\right)$ holds, and let $(x(t), v(t), \theta(t))$ be the forward characteristics (4.10). Then, there exists a positive constant $\tau>0$ such that

$$
\left|x(t)-x_{c}(t)\right|^{2}+\left|v(t)-v_{c}(t)\right|^{2}<\frac{R^{2}}{4} e^{-\frac{\kappa_{1} \psi(R)}{12\left(\theta_{c}^{0}+2 \varepsilon_{0}\right)} t}, \quad t \in[0, \tau] .
$$

Proof. We split the derivation of (4.16) into several steps.

Step A. (Derivation of time derivative for $\left.\frac{1}{2}|\hat{x}|^{2}+\frac{1}{2}|\hat{v}|^{2}+\lambda \hat{x} \cdot \hat{v}\right)$. We combine (4.13)(4.15) and set

$$
\lambda=\frac{\kappa_{1} \psi(R)}{8\left(\theta_{c}^{0}+2 \varepsilon_{0}\right)}<\frac{1}{2}
$$

to get

$$
\begin{aligned}
& \frac{d}{d s}\left(\frac{1}{2}|\hat{x}|^{2}+\frac{1}{2}|\hat{v}|^{2}+\lambda \hat{x} \cdot \hat{v}\right) \\
=- & \frac{\kappa_{1} \psi\left(d_{x}[f]\right)}{\theta_{c}^{0}+2 \varepsilon_{0}}|\hat{v}|^{2}+\frac{\kappa_{1}}{2\left(\theta_{c}^{0}-2 \varepsilon_{0}\right)}\left(|\hat{v}|^{2}+\mathcal{V}_{\omega}[f]\right) \\
& +\lambda|\hat{v}|^{2}+\lambda \frac{\kappa_{1}}{2\left(\theta_{c}^{0}-2 \varepsilon_{0}\right)}\left(2|\hat{x}|^{2}+|\hat{v}|^{2}+\mathcal{V}_{\omega}[f]\right)-\lambda|\hat{x}|^{2} \\
\leq- & \left(\frac{\kappa_{1} \psi\left(d_{x}[f]\right)}{\theta_{c}^{0}+2 \varepsilon_{0}}-\frac{\kappa_{1}}{2\left(\theta_{c}^{0}-2 \varepsilon_{0}\right)}-\lambda-\frac{\lambda \kappa_{1}}{2\left(\theta_{c}^{0}-2 \varepsilon_{0}\right)}\right)|\hat{v}(s)|^{2} \\
& -\left(\lambda-\frac{\lambda \kappa_{1}}{\theta_{c}^{0}-2 \varepsilon_{0}}\right)|\hat{x}(s)|^{2}+\left(\frac{\kappa_{1}}{2\left(\theta_{c}^{0}-2 \varepsilon_{0}\right)}+\lambda \frac{\kappa_{1}}{2\left(\theta_{c}^{0}-2 \varepsilon_{0}\right)}\right) \mathcal{V}_{\omega}[f] \\
\leq- & \frac{\kappa_{1} \psi(R)}{16\left(\theta_{c}^{0}+2 \varepsilon_{0}\right)}\left(|\hat{v}|^{2}+|\hat{x}|^{2}\right)+C e^{-\frac{\kappa_{1} \psi(R)}{6\left(\theta_{c}^{0}+2 \varepsilon_{0}\right)} s},
\end{aligned}
$$

where we used the relation (1) in (3.6) and Proposition 4.1

$$
\begin{aligned}
& C:=\frac{2(\lambda+1) \kappa_{1} \mathcal{V}_{\omega}^{0}}{\theta_{c}^{0}-2 \varepsilon_{0}}, \\
& \frac{2}{3} \frac{\kappa_{1}}{\theta_{c}^{0}-2 \varepsilon_{0}}<\frac{\kappa_{1} \psi(R)}{\theta_{c}^{0}+2 \varepsilon_{0}} \leq \frac{\kappa_{1}}{\theta_{c}^{0}-2 \varepsilon_{0}}<\frac{1}{2}, \\
& \mathcal{V}_{\omega}[f](t) \leq 4 \mathcal{V}_{\omega}^{0} e^{-\frac{\kappa_{1} \psi(R)}{6\left(\theta_{c}^{0}+2 \varepsilon_{0}\right)} t} .
\end{aligned}
$$


Step B. (Decay estimate for $\left.\frac{1}{2}|\hat{x}|^{2}+\frac{1}{2}|\hat{v}|^{2}+\lambda \hat{x} \cdot \hat{v}\right)$. Note that

$$
\frac{3}{16}\left(|\hat{x}|^{2}+|\hat{v}|^{2}\right) \leq \frac{1}{2}|\hat{x}|^{2}+\frac{1}{2}|\hat{v}|^{2}+\lambda \hat{x} \cdot \hat{v} \leq \frac{3}{4}\left(|\hat{x}|^{2}+|\hat{v}|^{2}\right) .
$$

Now, we combine (4.17) and (4.18) to get

$$
\begin{aligned}
& \frac{d}{d s}\left(\frac{1}{2}|\hat{x}|^{2}+\frac{1}{2}|\hat{v}|^{2}+\lambda \hat{x} \cdot \hat{v}\right) \\
\leq & -\frac{\kappa_{1} \psi(R)}{12\left(\theta_{c}^{0}+2 \varepsilon_{0}\right)}\left(\frac{1}{2}|\hat{x}|^{2}+\frac{1}{2}|\hat{v}|^{2}+\lambda \hat{x} \cdot \hat{v}\right)+C e^{-\frac{\kappa_{1} \psi(R)}{6\left(\theta_{c}^{0}+2 \varepsilon_{0}\right)} s} .
\end{aligned}
$$

Then, we apply Lemma 2.3 to obtain

$$
\begin{gathered}
\quad \frac{1}{2}|\hat{x}(t)|^{2}+\frac{1}{2}|\hat{v}(t)|^{2}+\lambda \hat{x}(t) \cdot \hat{v}(t) \\
\leq e^{-\frac{\kappa_{1} \psi(R)}{12\left(\theta_{c}^{0}+2 \varepsilon_{0}\right)} t}\left(\frac{1}{2}|\hat{x}(0)|^{2}+\frac{1}{2}|\hat{v}(0)|^{2}+\lambda \hat{x}(0) \cdot \hat{v}(0)\right) \\
-\frac{C}{\frac{\kappa_{1} \psi(R)}{12\left(\theta_{c}^{0}+2 \varepsilon_{0}\right)}}\left(e^{-\frac{\kappa_{1} \psi(R)}{6\left(\theta_{c}^{0}+2 \varepsilon_{0}\right)} t}-e^{-\frac{\kappa_{1} \psi(R)}{12\left(\theta_{c}^{0}+2 \varepsilon_{0}\right)} t}\right) .
\end{gathered}
$$

Step C. (Decay estimate for $|\hat{x}|^{2}+|\hat{v}|^{2}$ ). Note that

$$
\begin{aligned}
\frac{12\left(\theta_{c}^{0}+2 \varepsilon_{0}\right) C}{\kappa_{1} \psi(R)} & =\frac{\kappa_{1}}{\theta_{c}^{0}-2 \varepsilon_{0}} \frac{24(1+\lambda)\left(\theta_{c}^{0}+2 \varepsilon_{0}\right)}{\kappa_{1} \psi(R)} \mathcal{V}_{\omega}^{0} \\
& \leq 36 \frac{\theta_{c}^{0}+2 \varepsilon_{0}}{\psi(R)\left(\theta_{c}^{0}-2 \varepsilon_{0}\right)} \mathcal{V}_{\omega}^{0}=\frac{3}{16} K \mathcal{V}_{\omega}^{0}
\end{aligned}
$$

We use (4.18), (4.19) and $\mathcal{V}_{\omega}^{0}<\frac{R^{2}}{8 K}$ to get

$$
\begin{aligned}
|\hat{x}(s)|^{2}+|\hat{v}(s)|^{2} & \leq e^{-\frac{\kappa_{1} \psi(R)}{12\left(\theta_{c}^{0}+2 \varepsilon_{0}\right)} t}\left(4|\hat{x}(0)|^{2}+4|\hat{v}(0)|^{2}+K \mathcal{V}_{\omega}^{0}\right) \\
& <\frac{R^{2}}{2 K}+\frac{R^{2}}{8}<\frac{3 R^{2}}{16} .
\end{aligned}
$$

Hence we improve the second ansatz (3.5) to conclude

$$
\left|x(t)-x_{c}(t)\right|<\frac{R}{2}, \quad\left|v(t)-v_{c}(t)\right|<\frac{R}{2}, \quad t \in\left[0, \tau^{*}\right] .
$$


Step D. (Extension of $\tau^{*}$ ). We claim that $\tau^{*}$ in $(4.20)$ can be extended to $\tau$

$$
\tau^{*}=\tau
$$

If $\tau^{*}<\tau$, then there exists some $\tau_{1} \in\left(\tau^{*}, \tau\right)$ such that either $\hat{x}\left(\tau_{1}\right)=R / 2$ or $\hat{v}\left(\tau_{1}\right)=$ $R / 2$. Then we repeat the proofs in Propositions 4.1 and 4.2 to find

$$
\begin{aligned}
\frac{R^{2}}{4} & \leq\left|\hat{x}\left(\tau_{1}\right)\right|^{2}+\left|\hat{v}\left(\tau_{1}\right)\right|^{2} \\
& \leq e^{-\frac{\kappa_{1} \psi(R)}{12\left(\theta_{c}^{0}+2 \varepsilon_{0}\right)} \tau_{1}}\left(4|\hat{x}(0)|^{2}+4|\hat{v}(0)|^{2}+K \mathcal{V}_{\omega}^{0}\right) \\
& <\frac{R^{2}}{2 K}+\frac{R^{2}}{8}<\frac{3 R^{2}}{16}
\end{aligned}
$$

which yields a contradiction. Hence we close the ansatz (3.5) for $t \in[0, \tau]$.

\section{Concentration of temperature}

In this section, we provide the concentration estimate of temperature around the average value via a variance functional in a small-time interval to improve the first ansatz (3.3).

\subsection{Decay estimate of variance functional in temperature variable}

In this subsection, we present the exponential decay estimate of the variance functional measuring the temperature fluctuation in a small-time interval.

Proposition 5.1. Suppose the framework $\left(\mathcal{F}_{A}\right)-\left(\mathcal{F}_{C}\right)$ holds, and let $f$ be a classical solution to (1.3) which decays to zero at infinity. Then, there exists some positive constant $\tau>0$ such that for $t \in[0, \tau]$

$$
\int_{\Omega}\left|\theta-\theta_{c}(t)\right|^{2} f d z \leq \frac{\varepsilon_{0}^{2}}{2 M} e^{-\frac{\kappa_{1} \psi(R)}{6\left(\theta_{c}^{0}+2 \varepsilon_{0}\right)} t} .
$$

Proof. It follows from Propositions 4.1, 4.2 and ansatz (3.3) that there exists a positive constant $\tau$ such that for $t \in[0, \tau]$,

$$
\cup_{0 \leq t \leq \tau} \Omega_{\theta}(f, t) \subseteq\left(\theta_{c}^{0}-2 \varepsilon_{0}, \theta_{c}^{0}+2 \varepsilon_{0}\right),
$$




$$
\begin{aligned}
& \mathcal{V}_{\omega}[f](t) \leq 4 \mathcal{V}_{\omega}^{0} e^{-\frac{\kappa_{1} \psi(R)}{6\left(\theta_{c}^{0}+2 \varepsilon_{0}\right)} t}, \\
& \left|x(t)-x_{c}(t)\right|^{2}+\left|v(t)-v_{c}(t)\right|^{2}<\frac{R^{2}}{4} e^{-\frac{\kappa_{1} \psi(R)}{12\left(\theta_{c}^{0}+2 \varepsilon_{0}\right)} t}
\end{aligned}
$$

Then we have

$$
\begin{aligned}
\frac{d}{d t} \int_{\Omega}\left|\theta-\theta_{c}(t)\right|^{2} f d z & =-2 \int_{\Omega}\left(\theta-\theta_{c}\right) \frac{d \theta_{c}}{d t} f d z+\int_{\Omega}\left|\theta-\theta_{c}\right|^{2} \partial_{t} f d z \\
& =: \mathcal{I}_{21}+\mathcal{I}_{22}
\end{aligned}
$$

In what follows, we estimate the term $\mathcal{I}_{2 i}, i=1,2$ one by one.

- (Estimate of $\left.\mathcal{I}_{21}\right)$. By definition of $\theta_{c}$, one has

$$
\mathcal{I}_{21}=-2 \int_{\Omega}\left(\theta-\theta_{c}\right) \frac{d \theta_{c}}{d t} f d z=-2 \frac{d \theta_{c}}{d t} \int_{\Omega}\left(\theta-\theta_{c}\right) f d z=0 .
$$

- (Estimate of $\left.\mathcal{I}_{22}\right)$. We use (1.3a) to see

$$
\begin{aligned}
\mathcal{I}_{22}= & -\int_{\Omega}\left|\theta-\theta_{\mathcal{c}}(t)\right|^{2} \nabla_{x} \cdot(v f) d z-\int_{\Omega}\left|\theta-\theta_{c}(t)\right|^{2} \nabla_{v} \cdot(\mathcal{F}[f] f) d z \\
& -\int_{\Omega}\left|\theta-\theta_{\mathcal{c}}(t)\right|^{2} \partial_{\theta}(\mathcal{G}[f] f) d z \\
= & : \mathcal{I}_{221}+\mathcal{I}_{222}+\mathcal{I}_{223} .
\end{aligned}
$$

- (Estimate of $\mathcal{I}_{22 i}, i=1,2$ ). It is easy to see

$$
\mathcal{I}_{221}=0, \quad \mathcal{I}_{222}=0 \text {. }
$$

- (Estimate of $\left.\mathcal{I}_{223}\right)$. By direct calculation, one has

$$
\begin{aligned}
\mathcal{I}_{223}= & 2 \int_{\Omega}\left(\theta-\theta_{\mathcal{C}}(t)\right) \mathcal{G}[f] f d z \\
= & 2 \kappa_{1} \int_{\Omega^{2}} \psi\left(\left|x-x_{*}\right|\right)\left(\theta-\theta_{\mathcal{C}}\right)\left(v-v_{\mathcal{C}}\right) \cdot\left(\frac{v-v_{\mathcal{C}}}{\theta}-\frac{v_{*}-v_{\mathcal{C}}}{\theta_{*}}\right) f_{*} f d z_{*} d z \\
& +2 \kappa_{2} \int_{\Omega^{2}} \zeta\left(\left|x-x_{*}\right|\right)\left(\theta-\theta_{\mathcal{C}}\right)\left(\frac{1}{\theta}-\frac{1}{\theta_{*}}\right) f_{*} f d z_{*} d z+2 \int_{\Omega}\left(\theta-\theta_{\mathcal{C}}\right) x \cdot v f d z \\
= & : \mathcal{I}_{2231}+\mathcal{I}_{2232}+\mathcal{I}_{2233} .
\end{aligned}
$$

Below, we estimate the term $\mathcal{I}_{223 i}$ separately. 
- (Estimate for $\left.\mathcal{I}_{2231}\right)$. By direct estimate, one has

$$
\begin{aligned}
\mathcal{I}_{2231} & =2 \kappa_{1} \int_{\Omega^{2}} \psi\left(\left|x-x_{*}\right|\right)\left(\theta-\theta_{c}\right)\left(v-v_{\mathcal{C}}\right) \cdot\left(\frac{v-v_{\mathcal{C}}}{\theta}-\frac{v_{*}-v_{\mathcal{C}}}{\theta_{*}}\right) f_{*} f d z_{*} d z \\
& \leq \frac{R \kappa_{1}}{\theta_{c}^{0}-2 \varepsilon_{0}} \int_{\Omega}\left(\left|\theta-\theta_{c}\right|^{2}+\left|v-v_{\mathcal{C}}\right|^{2}\right) f d z \\
& \leq \frac{R \kappa_{1}}{\theta_{c}^{0}-2 \varepsilon_{0}} \int_{\Omega}\left|\theta-\theta_{c}\right|^{2} f d z+\frac{R \kappa_{1}}{\theta_{c}^{0}-2 \varepsilon_{0}} \int_{\Omega}\left|v-v_{\mathcal{C}}\right|^{2} f d z .
\end{aligned}
$$

- (Estimate for $\mathcal{I}_{2232}$ ). We have

$$
\begin{aligned}
\mathcal{I}_{2232} & =\kappa_{2} \int_{\Omega^{2}} \zeta\left(\left|x-x_{*}\right|\right)\left(\theta-\theta_{*}\right)\left(\frac{1}{\theta}-\frac{1}{\theta_{*}}\right) f_{*} f d z_{*} d z \\
& =-\kappa_{2} \int_{\Omega^{2}} \zeta\left(\left|x-x_{*}\right|\right) \frac{\left|\theta-\theta_{*}\right|^{2}}{\theta \theta_{*}} f_{*} f d z_{*} d z \\
& \leq-\frac{\kappa_{2} \zeta(R)}{\left(\theta_{c}^{0}+2 \varepsilon_{0}\right)^{2}} \int_{\Omega^{2}}\left|\theta-\theta_{*}\right|^{2} f_{*} f d z_{*} d z \\
& =-\frac{2 \kappa_{2} \zeta(R)}{\left(\theta_{c}^{0}+2 \varepsilon_{0}\right)^{2}} \int_{\Omega}\left|\theta-\theta_{\mathcal{C}}\right|^{2} f d z .
\end{aligned}
$$

- (Estimate for $\mathcal{I}_{2233}$ ). Similarly, one has

$$
\begin{aligned}
\mathcal{I}_{2233}= & 2 \int_{\Omega}\left(\theta-\theta_{c}\right) x \cdot v f d z \\
= & 2\left[\int_{\Omega}\left(\theta-\theta_{c}\right)\left(x-x_{c}\right) \cdot\left(v-v_{c}\right) f d z+v_{\mathcal{c}} \cdot \int_{\Omega}\left(\theta-\theta_{c}\right)\left(x-x_{c}\right) f d z\right. \\
& \left.+x_{c} \cdot \int_{\Omega}\left(\theta-\theta_{c}\right)\left(v-v_{c}\right) f d z\right] \\
\leq & \frac{R}{2} \int_{\Omega}\left(\left|\theta-\theta_{c}\right|^{2}+\left|x-x_{c}\right|^{2}\right) f d z+\left|v_{\mathcal{c}}\right| \int_{\Omega}\left(\left|\theta-\theta_{c}\right|^{2}+\left|x-x_{c}\right|^{2}\right) f d z \\
& +\left|x_{c}\right| \int_{\Omega}\left(\left|\theta-\theta_{c}\right|^{2}+\left|v-v_{\mathcal{c}}\right|^{2}\right) f d z \\
\leq & \left(\frac{R}{2}+2 \sqrt{2}\left|\omega_{c}^{0}\right|\right) \int_{\Omega}\left|\theta-\theta_{c}\right|^{2} f d z+\left(\frac{R}{2}+\sqrt{2}\left|\omega_{c}^{0}\right|\right) \int_{\Omega}\left|x-x_{c}\right|^{2} f d z \\
& +\sqrt{2}\left|\omega_{c}^{0}\right| \int_{\Omega}\left(v-v_{c}\right)^{2} f d z .
\end{aligned}
$$

In (5.1), we combine (5.2)-(5.3) together to derive a differential inequality $\frac{d}{d t} \int_{\Omega}\left|\theta-\theta_{c}\right|^{2} f d z \leq-\left[\frac{2 \kappa_{2} \zeta(R)}{\left(\theta_{c}^{0}+2 \varepsilon_{0}\right)^{2}}-\frac{R \kappa_{1}}{\theta_{c}^{0}-2 \varepsilon_{0}}-\left(\frac{R}{2}+2 \sqrt{2}\left|\omega_{c}^{0}\right|\right)\right] \int_{\Omega}\left|\theta-\theta_{c}\right|^{2} f d z$ 


$$
\begin{aligned}
& +\left(\frac{R \kappa_{1}}{\theta_{c}^{0}-2 \varepsilon_{0}}+\sqrt{2}\left|\omega_{c}^{0}\right|\right) \int_{\Omega}\left|v-v_{c}\right|^{2} f d z+\left(\frac{R}{2}+\sqrt{2}\left|\omega_{c}^{0}\right|\right) \int_{\Omega}\left|x-x_{c}\right|^{2} f d z \\
\leq & -\frac{\kappa_{2} \zeta(R)}{\left(\theta_{c}^{0}+2 \varepsilon_{0}\right)^{2}} \int_{\Omega}\left|\theta-\theta_{c}\right|^{2} f d z+\left(\frac{R \kappa_{1}}{\theta_{c}^{0}-2 \varepsilon_{0}}+\frac{R}{2}+\sqrt{2}\left|\omega_{c}^{0}\right|\right) \mathcal{V}_{\omega}[f] \\
\leq & \frac{-\kappa_{2} \zeta(R)}{\left(\theta_{c}^{0}+2 \varepsilon_{0}\right)^{2}} \int_{\Omega}\left|\theta-\theta_{c}\right|^{2} f d z+4\left(\frac{R \kappa_{1}}{\theta_{c}^{0}-2 \varepsilon_{0}}+\frac{R}{2}+\sqrt{2}\left|\omega_{c}^{0}\right|\right) \mathcal{V}_{\omega}^{0} e^{-\frac{\kappa_{1} \psi(R)}{6\left(\theta_{c}^{0}+2 \varepsilon_{0}\right)} t},
\end{aligned}
$$

where we used the relation (2) in (3.6)

$$
\frac{\kappa_{2} \zeta(R)}{\left(\theta_{c}^{0}+2 \varepsilon_{0}\right)^{2}}>\frac{R \kappa_{1}}{\theta_{c}^{0}-2 \varepsilon_{0}}+\left(\frac{R}{2}+2 \sqrt{2}\left|\omega_{c}^{0}\right|\right) .
$$

We apply Lemma 2.3 to the differential inequality (5.4) to get the desired result

$$
\begin{aligned}
& \int_{\Omega}\left|\theta-\theta_{c}\right|^{2} f d z \\
\leq & e^{-\frac{\kappa_{2} \zeta(R)}{\left(\theta_{c}^{0}+2 \varepsilon_{0}\right)^{2}} t} \int_{\Omega}\left|\theta-\theta_{c}(0)\right|^{2} f^{0} d z+\frac{4\left(\frac{R \kappa_{1}}{\theta_{c}^{0}-2 \varepsilon_{0}}+\frac{R}{2}+\sqrt{2}\left|\omega_{c}^{0}\right|\right) \mathcal{V}_{\omega}^{0}}{\frac{\kappa_{2} \zeta(R)}{\left(\theta_{c}^{0}+2 \varepsilon_{0}\right)^{2}}-\frac{\kappa_{1} \psi(R)}{6\left(\theta_{c}^{0}+2 \varepsilon_{0}\right)}} \\
& \times\left(e^{-\frac{\kappa_{1} \psi(R)}{6\left(\theta_{c}^{0}+2 \varepsilon_{0}\right)} t}-e^{-\frac{\kappa_{2} \zeta(R)}{\left(\theta_{c}^{0}+2 \varepsilon_{0}\right)^{2}} t}\right) \\
\leq & e^{-\frac{\kappa_{1} \psi(R)}{6\left(\theta_{c}^{0}+2 \varepsilon_{0}\right)} t}\left[\int_{\Omega}\left|\theta-\theta_{c}(0)\right|^{2} f^{0} d z+24 \frac{\theta_{c}^{0}+2 \varepsilon_{0}}{\kappa_{1} \psi(R)}\left(\frac{R \kappa_{1}}{\theta_{c}^{0}-2 \varepsilon_{0}}+\frac{R}{2}+\sqrt{2}\left|\omega_{c}^{0}\right|\right) \mathcal{V}_{\omega}^{0}\right] \\
\leq & \frac{\varepsilon_{0}^{2}}{2 M} e^{-\frac{\kappa_{1} \psi(R)}{6\left(\theta_{c}^{0}+2 \varepsilon_{0}\right)} t},
\end{aligned}
$$

where we used the relations (3) and (4) in (3.6)

$$
\begin{aligned}
& \frac{\kappa_{2} \zeta(R)}{\left(\theta_{c}^{0}+2 \varepsilon_{0}\right)^{2}} \geq \frac{\kappa_{1}}{3\left(\theta_{c}^{0}-2 \varepsilon_{0}\right)} \geq \frac{\kappa_{1} \psi(R)}{3\left(\theta_{c}^{0}+2 \varepsilon_{0}\right)} \\
& 24 \frac{\theta_{c}^{0}+2 \varepsilon_{0}}{\kappa_{1} \psi(R)}\left(\frac{R \kappa_{1}}{\theta_{c}^{0}-2 \varepsilon_{0}}+\frac{R}{2}+\sqrt{2}\left|\omega_{c}^{0}\right|\right) \mathcal{V}_{\omega}^{0} \leq \frac{\varepsilon_{0}^{2}}{4 M}
\end{aligned}
$$

Then we finish the proof of this proposition.

\subsection{Pointwise exponential concentration of temperature variable}

In this subsection, we study pointwise decay estimate of temperature fluctuation around the average temperature along the forward characteristics. 
Proposition 5.2. Suppose the framework $\left(\mathcal{F}_{A}\right)-\left(\mathcal{F}_{C}\right)$ holds, and let $f$ be the global classical solution to (1.3). Then, there exists some positive constant $\tau>0$ such that for $t \in[0, \tau]$

$$
\left|\theta(t)-\theta_{c}(t)\right|^{2}<\frac{\varepsilon_{0}^{2}}{2} e^{-\frac{\kappa_{1} \psi(R)}{12\left(\theta_{c}^{0}+2 \varepsilon_{0}\right)} t}
$$

Proof. By Proposition 4.1, 4.2 and 5.1, there exists a positive constant $\tau>0$ such that for $t \in[0, \tau]$,

$$
\begin{aligned}
& \cup_{\tau \geq t \geq 0} \Omega_{\theta}(f, t) \subseteq\left(\theta_{c}^{0}-2 \varepsilon_{0}, \theta_{c}^{0}+2 \varepsilon_{0}\right) \\
& \int_{\Omega}\left|\theta-\theta_{c}\right|^{2} f d z \leq \frac{\varepsilon_{0}^{2}}{2 M} e^{-\frac{\kappa_{1} \psi(R)}{6\left(\theta_{c}^{0}+2 \varepsilon_{0}\right)} t} \\
& \mathcal{V}_{\omega}[f](t) \leq 4 e^{-\frac{\kappa_{1} \psi(R)}{6\left(\theta_{c}^{0}+2 \varepsilon_{0}\right)} t} \mathcal{V}_{\omega}^{0} \\
& |\hat{x}(s)|^{2}+|\hat{v}(s)|^{2}<\frac{R^{2}}{4} e^{-\frac{\kappa_{1} \psi(R)}{12\left(\theta_{c}^{0}+2 \varepsilon_{0}\right)} t} \\
& d_{x}[f(t)]<R, \quad d_{v}[f(t)]<R .
\end{aligned}
$$

Then, along the characteristics, we have

$$
\begin{aligned}
\frac{d}{d s}\left|\theta-\theta_{\mathcal{C}}\right|^{2}= & 2\left(\theta-\theta_{\mathcal{c}}\right)\left(\mathcal{G}[f]-\int_{\Omega} \mathcal{G}[f] f d z\right) \\
= & 2 \kappa_{2}\left(\theta-\theta_{c}\right) \int_{\Omega} \zeta\left(\left|x-x_{*}\right|\right)\left(\frac{1}{\theta}-\frac{1}{\theta_{*}}\right) f_{*} d z_{*} \\
& +2 \kappa_{1}\left(\theta-\theta_{c}\right) \int_{\Omega} \psi\left(\left|x-x_{*}\right|\right)\left(v-v_{\mathcal{C}}\right) \cdot\left(\frac{v-v_{\mathcal{C}}}{\theta}-\frac{v_{*}-v_{\mathcal{c}}}{\theta_{*}}\right) f_{*} d z_{*} \\
& -2 \kappa_{1}\left(\theta-\theta_{\mathcal{C}}\right) \int_{\Omega^{2}} \psi\left(\left|x-x_{*}\right|\right)\left(v-v_{\mathcal{C}}\right) \cdot\left(\frac{v-v_{\mathcal{C}}}{\theta}-\frac{v_{*}-v_{\mathcal{C}}}{\theta_{*}}\right) f_{*} f d z_{*} d z \\
& +2\left(\theta-\theta_{\mathcal{C}}\right)\left(x \cdot v-\int_{\Omega} x \cdot v f d z\right) \\
= & : \mathcal{I}_{31}+\mathcal{I}_{32}+\mathcal{I}_{33}+\mathcal{I}_{34} .
\end{aligned}
$$

In what follows, we provide estimates for $\mathcal{I}_{3 i}, i=1, \ldots, 4$ one by one.

- (Estimate for $\left.\mathcal{I}_{31}\right)$. By direct estimate, one has

$$
\mathcal{I}_{31}=2 \kappa_{2}\left(\theta-\theta_{c}\right) \int_{\Omega} \zeta\left(\left|x-x_{*}\right|\right)\left(\frac{1}{\theta}-\frac{1}{\theta_{*}}\right) f_{*} d z_{*}
$$




$$
\begin{aligned}
& =2 \kappa_{2}\left(\theta-\theta_{c}\right) \int_{\Omega} \zeta\left(\left|x-x_{*}\right|\right) \frac{\left(\theta_{*}-\theta_{c}\right)-\left(\theta-\theta_{c}\right)}{\theta_{*} \theta} f_{*} d z_{*} \\
& =-2 \kappa_{2} \int_{\Omega} \zeta\left(\left|x-x_{*}\right|\right) \frac{\left(\theta-\theta_{c}\right)^{2}-\left(\theta-\theta_{c}\right)\left(\theta_{*}-\theta_{c}\right)}{\theta_{*} \theta} f_{*} d z_{*} \\
& \leq-2 \kappa_{2}\left(\theta-\theta_{c}\right)^{2} \frac{\zeta(R)}{\left(\theta_{c}^{0}+2 \varepsilon_{0}\right)^{2}}+\frac{\kappa_{2}}{\left(\theta_{c}^{0}-2 \varepsilon_{0}\right)^{2}} \int_{\Omega}\left(\left(\theta-\theta_{c}\right)^{2}+\left(\theta_{*}-\theta_{c}\right)^{2}\right) f_{*} d z_{*} \\
& \leq-\left(\frac{2 \kappa_{2} \zeta(R)}{\left(\theta_{c}^{0}+2 \varepsilon_{0}\right)^{2}}-\frac{\kappa_{2}}{\left(\theta_{c}^{0}-2 \varepsilon_{0}\right)^{2}}\right)\left(\theta-\theta_{c}\right)^{2}+\frac{\kappa_{2}}{\left(\theta_{c}^{0}-2 \varepsilon_{0}\right)^{2}} \int_{\Omega}\left(\theta_{*}-\theta_{c}\right)^{2} f_{*} d z_{*} \\
& \leq-\left(\frac{2 \kappa_{2} \zeta(R)}{\left(\theta_{c}^{0}+2 \varepsilon_{0}\right)^{2}}-\frac{\kappa_{2}}{\left(\theta_{c}^{0}-2 \varepsilon_{0}\right)^{2}}\right)\left(\theta-\theta_{c}\right)^{2}+\frac{\kappa_{2}}{\left(\theta_{c}^{0}-2 \varepsilon_{0}\right)^{2}} \frac{\varepsilon_{0}^{2}}{2 M} e^{-\frac{\kappa_{1} \psi(R)}{6\left(\theta_{c}^{0}+2 \varepsilon_{0}\right)} t} \\
& \leq-\frac{2 \kappa_{2} \zeta(R)}{3\left(\theta_{c}^{0}+2 \varepsilon_{0}\right)^{2}}\left(\theta-\theta_{c}\right)^{2}+\frac{\kappa_{2}}{\left(\theta_{c}^{0}-2 \varepsilon_{0}\right)^{2}} \frac{\varepsilon_{0}^{2}}{2 M} e^{-\frac{\kappa_{1} \psi(R)}{6\left(\theta_{c}^{0}+2 \varepsilon_{0}\right)} t}
\end{aligned}
$$

where we used the relation (5) in (3.6)

$$
\frac{4}{3} \frac{\zeta(R)}{\left(\theta_{c}^{0}+2 \varepsilon_{0}\right)^{2}}>\frac{1}{\left(\theta_{c}^{0}-2 \varepsilon_{0}\right)^{2}} .
$$

- (Estimate for $\left.\mathcal{I}_{32}\right)$. One has

$$
\begin{aligned}
\mathcal{I}_{32} & =2 \kappa_{1}\left(\theta-\theta_{c}\right) \int_{\Omega} \psi\left(\left|x-x_{*}\right|\right)\left(v-v_{c}\right) \cdot\left(\frac{v-v_{c}}{\theta}-\frac{v_{*}-v_{c}}{\theta_{*}}\right) f_{*} d z_{*} \\
\leq & \frac{2 \kappa_{1}}{\theta_{c}^{0}-2 \varepsilon_{0}}\left|\theta-\theta_{c}\right|\left|v-v_{c}\right|^{2}+\frac{\kappa_{1}}{\theta_{c}^{0}-2 \varepsilon_{0}}\left|\theta-\theta_{c}\right| \int_{\Omega}\left(\left(v-v_{c}\right)^{2}+\left(v_{*}-v_{c}\right)^{2}\right) f_{*} d z_{*} \\
\leq & \frac{\kappa_{1}}{\theta_{c}^{0}-2 \varepsilon_{0}}\left|\theta-\theta_{c}\right|^{2}+\frac{\kappa_{1}}{\theta_{c}^{0}-2 \varepsilon_{0}}\left|v-v_{c}\right|^{4}+\frac{\kappa_{1}}{\theta_{c}^{0}-2 \varepsilon_{0}}\left|\theta-\theta_{c}\right|^{2} \\
& +\frac{\kappa_{1}}{2\left(\theta_{c}^{0}-2 \varepsilon_{0}\right)}\left|v-v_{c}\right|^{4}+\frac{\kappa_{1}}{2\left(\theta_{c}^{0}-2 \varepsilon_{0}\right)}\left(\int_{\Omega}\left|v_{*}-v_{c}\right|^{2} f_{*} d z_{*}\right)^{2} \\
\leq & \frac{2 \kappa_{1}}{\theta_{c}^{0}-2 \varepsilon_{0}}\left|\theta-\theta_{c}\right|^{2}+\frac{3 \kappa_{1}}{2\left(\theta_{c}^{0}-2 \varepsilon_{0}\right)}\left|v-v_{c}\right|^{4}+\frac{\kappa_{1}}{2\left(\theta_{c}^{0}-2 \varepsilon_{0}\right)}\left(\int_{\Omega}\left|v_{*}-v_{c}\right|^{2} f_{*} d z_{*}\right)^{2} \\
\leq & \frac{2 \kappa_{1}}{\theta_{c}^{0}-2 \varepsilon_{0}}\left|\theta-\theta_{c}\right|^{2}+\frac{\kappa_{1}}{2\left(\theta_{c}^{0}-2 \varepsilon_{0}\right)}\left(\frac{3 R^{4}}{16}+16\left|\mathcal{V}_{\omega}^{0}\right|^{2}\right) e^{-\frac{\kappa_{1} \psi(R)}{6\left(\theta_{c}^{0}+2 \varepsilon_{0}\right)}} t
\end{aligned}
$$

- (Estimate for $\left.\mathcal{I}_{33}\right)$. By direct calculation, one has

$$
\mathcal{I}_{33}=-2 \kappa_{1}\left(\theta-\theta_{c}\right) \int_{\Omega^{2}} \psi\left(\left|x-x_{*}\right|\right)\left(v-v_{\mathcal{C}}\right) \cdot\left(\frac{v-v_{c}}{\theta}-\frac{v_{*}-v_{c}}{\theta_{*}}\right) f_{*} f d z_{*} d z
$$




$$
\begin{aligned}
& \leq \frac{2 \kappa_{1}\left|\theta-\theta_{c}\right|}{\theta_{c}^{0}-2 \varepsilon_{0}} \int_{\Omega}\left|v-v_{\mathcal{C}}\right|^{2} f d z+\frac{2 \kappa_{1}\left|\theta-\theta_{c}\right|}{\theta_{c}^{0}-2 \varepsilon_{0}} \int_{\Omega}\left|v_{*}-v_{\mathcal{C}}\right|^{2} f_{*} d z_{*} \\
& \leq \frac{2 \kappa_{1}}{\theta_{c}^{0}-2 \varepsilon_{0}}\left|\theta-\theta_{c}\right|^{2}+\frac{2 \kappa_{1}}{\theta_{c}^{0}-2 \varepsilon_{0}}\left(\int_{\Omega}\left|v-v_{c}\right|^{2} f d z\right)^{2} \\
& \leq \frac{2 \kappa_{1}}{\theta_{c}^{0}-2 \varepsilon_{0}}\left|\theta-\theta_{c}\right|^{2}+\frac{2 \kappa_{1}}{\theta_{c}^{0}-2 \varepsilon_{0}} 16\left|\mathcal{V}_{\omega}^{0}\right|^{2} e^{-\frac{\kappa_{1} \psi(R)}{3\left(\theta_{c}^{0}+2 \varepsilon_{0}\right)} t} .
\end{aligned}
$$

- (Estimate for $\left.\mathcal{I}_{34}\right)$. Similarly, one has

$$
\begin{aligned}
& \mathcal{I}_{34}=2\left(\theta-\theta_{c}\right)\left(x \cdot v-\int_{\Omega} x \cdot v f d z\right) \\
& =2\left(\theta-\theta_{c}\right)\left(x \cdot v-\int_{\Omega}\left(x-x_{c}\right) \cdot\left(v-v_{c}\right) f d z-x_{c} \cdot v_{c}\right) \\
& \leq 2\left|x-x_{c}\right|\left|\theta-\theta_{c}\right|\left(\int_{\Omega}\left|v-v_{c}\right|^{2} f d z\right)^{\frac{1}{2}}+2\left(\theta-\theta_{c}\right)\left(v \cdot\left(x-x_{c}\right)+x_{c} \cdot\left(v-v_{c}\right)\right) \\
& \leq R\left(\frac{\left|\theta-\theta_{c}\right|^{2}}{2}+\frac{1}{2} \int_{\Omega}\left|v-v_{\mathcal{C}}\right|^{2} f d z\right)+|v|\left(\left|\theta-\theta_{\mathcal{C}}\right|^{2}+\left|x-x_{\mathcal{c}}\right|^{2}\right) \\
& +\left|x_{c}\right|\left(\left|\theta-\theta_{c}\right|^{2}+\left|v-v_{\mathcal{C}}\right|^{2}\right) \\
& \leq\left|\theta-\theta_{c}\right|^{2}\left(\frac{R}{2}+|v|+\left|x_{c}\right|\right)+\left|x_{c}\right|\left|v-v_{c}\right|^{2}+|v|\left|x-x_{c}\right|^{2}+\frac{R}{2} \int_{\Omega}\left|v-v_{c}\right|^{2} f d z \\
& \leq 2\left(\sqrt{2}\left|\omega_{c}^{0}\right|+\frac{R}{2}\right)\left|\theta-\theta_{c}\right|^{2}+\left(\sqrt{2}\left|\omega_{c}^{0}\right|+\frac{R}{2}\right)\left(\left|v-v_{c}\right|^{2}+\left|x-x_{c}\right|^{2}\right) \\
& +\frac{R}{2} \int_{\Omega}\left|v-v_{\mathcal{C}}\right|^{2} f d z \\
& \leq 2\left(\sqrt{2}\left|\omega_{c}^{0}\right|+\frac{R}{2}\right)\left|\theta-\theta_{c}\right|^{2}+\left(\sqrt{2}\left|\omega_{c}^{0}\right|+\frac{R}{2}\right) \frac{R^{2}}{4} e^{-\frac{\kappa_{1} \psi(R)}{12\left(\theta_{c}^{0}+2 \varepsilon_{0}\right)} t} \\
& +2 R \mathcal{V}_{\omega}^{0} e^{-\frac{\kappa_{1} \psi(R)}{6\left(\theta_{c}^{0}+2 \varepsilon_{0}\right)} t}
\end{aligned}
$$

Here we used Remark 2.2 to get

$$
|v| \leq\left|v-v_{c}\right|+\left|v_{c}\right| \leq \frac{R}{2}+\sqrt{2}\left|\omega_{c}^{0}\right|
$$

Now, we collect all the estimates in (5.5)-(5.8) to derive the following differential inequality:

$$
\frac{d}{d s}\left|\theta-\theta_{c}\right|^{2} \leq-2\left(\frac{\kappa_{2} \zeta(R)}{3\left(\theta_{c}^{0}+2 \varepsilon_{0}\right)^{2}}-\frac{2 \kappa_{1}}{\theta_{c}^{0}-2 \varepsilon_{0}}-\left(\sqrt{2}\left|\omega_{c}^{0}\right|+\frac{R}{2}\right)\right)\left|\theta-\theta_{c}\right|^{2}
$$




$$
\begin{aligned}
& +\frac{\kappa_{2} \varepsilon_{0}^{2}}{2 M\left(\theta_{c}^{0}-2 \varepsilon_{0}\right)^{2}} e^{-\frac{\kappa_{1} \psi(R)}{6\left(\theta_{c}^{0}+2 \varepsilon_{0}\right)} t}+\frac{\kappa_{1}}{2\left(\theta_{c}^{0}-2 \varepsilon_{0}\right)}\left(\frac{3 R^{4}}{16}+16\left|\mathcal{V}_{\omega}^{0}\right|^{2}\right) e^{-\frac{\kappa_{1} \psi(R)}{6\left(\theta_{c}^{0}+2 \varepsilon_{0}\right)} t} \\
& +\frac{32\left|\mathcal{V}_{\omega}^{0}\right|^{2} \kappa_{1}}{\theta_{c}^{0}-2 \varepsilon_{0}} e^{-\frac{\kappa_{1} \psi(R)}{3\left(\theta_{c}^{0}+2 \varepsilon_{0}\right)} t}+\left(\sqrt{2}\left|\omega_{c}^{0}\right|+\frac{R}{2}\right) \frac{R^{2}}{4} e^{-\frac{\kappa_{1} \psi(R)}{12\left(\theta_{c}^{0}+2 \varepsilon_{0}\right)} t}+2 R \mathcal{V}_{\omega}^{0} e^{-\frac{\kappa_{1} \psi(R)}{6\left(\theta_{c}^{0}+2 \varepsilon_{0}\right)} t} \\
& \leq-2\left[\frac{\kappa_{2} \zeta(R)}{3\left(\theta_{c}^{0}+2 \varepsilon_{0}\right)^{2}}-\frac{2 \kappa_{1}}{\theta_{c}^{0}-2 \varepsilon_{0}}-\left(\sqrt{2}\left|\omega_{c}^{0}\right|+\frac{R}{2}\right)\right]\left|\theta-\theta_{c}\right|^{2} \\
& +\frac{\varepsilon_{0}^{2}}{M} \frac{\kappa_{2}}{\left|\theta_{c}^{0}-2 \varepsilon_{0}\right|^{2}} e^{-\frac{\kappa_{1} \psi(R)}{12\left(\theta_{c}^{0}+2 \varepsilon_{0}\right)} t}
\end{aligned}
$$

where we used the relation (7) in (3.6)

$$
\begin{aligned}
& \frac{\kappa_{1}}{2\left(\theta_{c}^{0}-2 \varepsilon_{0}\right)}\left(\frac{3 R^{4}}{16}+16\left|\mathcal{V}_{\omega}^{0}\right|^{2}\right)+\frac{32\left|\mathcal{V}_{\omega}^{0}\right|^{2} \kappa_{1}}{\theta_{c}^{0}-2 \varepsilon_{0}}+\left(\sqrt{2}\left|\omega_{c}^{0}\right|+\frac{R}{2}\right) \frac{R^{2}}{4}+2 R \mathcal{V}_{\omega}^{0} \\
< & \frac{\kappa_{2}}{\left(\theta_{c}^{0}-2 \varepsilon_{0}\right)^{2}} \frac{\varepsilon_{0}^{2}}{2 M} .
\end{aligned}
$$

Next, we again use the relation (6) in (3.6)

$$
\frac{\kappa_{2} \zeta(R)}{6\left(\theta_{c}^{0}+2 \varepsilon_{0}\right)^{2}}>\frac{2 \kappa_{1}}{\theta_{c}^{0}-2 \varepsilon_{0}}+\left(\sqrt{2}\left|\omega_{c}^{0}\right|+\frac{R}{2}\right)
$$

to further simplify (5.9)

$$
\frac{d}{d s}\left|\theta-\theta_{c}\right|^{2} \leq-\frac{\kappa_{2} \zeta(R)}{3\left(\theta_{c}^{0}+2 \varepsilon_{0}\right)^{2}}\left|\theta-\theta_{\mathcal{c}}\right|^{2}+\frac{\varepsilon_{0}^{2}}{M} \frac{\kappa_{2}}{\left(\theta_{c}^{0}-2 \varepsilon_{0}\right)^{2}} e^{-\frac{\kappa_{1} \psi(R)}{12\left(\theta_{c}^{0}+2 \varepsilon_{0}\right)} t} .
$$

We apply Lemma 2.3 for (5.10) to get

$$
\begin{aligned}
\left|\theta-\theta_{c}\right|^{2} & \leq e^{-\frac{\kappa_{2} \zeta(R)}{3\left(\theta_{c}^{0}+2 \varepsilon_{0}\right)^{2}} t}\left|\theta-\theta_{c}(0)\right|^{2}+\frac{\frac{1}{M} \frac{\kappa_{2} \varepsilon_{0}^{2}}{\left(\theta_{c}^{0}-2 \varepsilon_{0}\right)^{2}}}{\frac{\kappa_{2} \zeta(R)}{3\left(\theta_{c}^{0}+2 \varepsilon_{0}\right)^{2}}-\frac{\kappa_{1} \psi(R)}{12\left(\theta_{c}^{0}+2 \varepsilon_{0}\right)}}\left(e^{-\frac{\kappa_{1} \psi(R)}{12\left(\theta_{c}^{0}+2 \varepsilon_{0}\right)} t}-e^{-\frac{\kappa_{2} \zeta(R)}{3\left(\theta_{c}^{0}+2 \varepsilon_{0}\right)^{2}} t}\right) \\
& \leq e^{-\frac{\kappa_{2} \zeta(R)}{3\left(\theta_{c}^{0}+2 \varepsilon_{0}\right)^{2}} t} \frac{\varepsilon_{0}^{2}}{4 M}+\frac{12}{M} \frac{\varepsilon_{0}^{2}}{\zeta(R)} \frac{\left(\theta_{c}^{0}+2 \varepsilon_{0}\right)^{2}}{\left(\theta_{c}^{0}-2 \varepsilon_{0}\right)^{2}} e^{-\frac{\kappa_{1} \psi(R)}{12\left(\theta_{c}^{0}+2 \varepsilon_{0}\right)} t} \\
& <\frac{\varepsilon_{0}^{2}}{2} e^{-\frac{\kappa_{1} \psi(R)}{12\left(\theta_{c}^{0}+2 \varepsilon_{0}\right)} t}
\end{aligned}
$$

where we used the relations (3) in (3.6)

$$
\frac{\kappa_{2} \zeta(R)}{\left(\theta_{c}^{0}+2 \varepsilon_{0}\right)^{2}} \geq \frac{\kappa_{1}}{3\left(\theta_{c}^{0}-2 \varepsilon_{0}\right)} \geq \frac{\kappa_{1} \psi(R)}{3\left(\theta_{c}^{0}+2 \varepsilon_{0}\right)}, \quad \frac{12}{M} \frac{1}{\zeta(R)} \frac{\left(\theta_{c}^{0}+2 \varepsilon_{0}\right)^{2}}{\left(\theta_{c}^{0}-2 \varepsilon_{0}\right)^{2}}=\frac{1}{4} .
$$

Hence we improve the first ansatz (3.3) and prove this proposition. 


\section{Proof of Theorem 3.1}

In this section, we provide a proof of Theorem 3.1. For this, it sufficies to show that the first ansatz (3.3) holds in the whole-time interval $[0, \infty)$. We define

$$
\mathcal{S}:=\left\{\tau>0: \cup_{0 \leq t \leq \tau} \Omega_{\theta}(f, t) \subseteq B_{\varepsilon_{0}}\left(\theta_{c}(t)\right), \theta_{c}^{0}-\varepsilon_{0}<\theta_{c}(t)<\theta_{c}^{0}+\varepsilon_{0} \text { for } t \in(0, \tau)\right\} .
$$

By the assumption (3.2) and the continuity of $\theta, \mathcal{S}$ is not empty and thus, we set its supremum $\tau^{*}$

$$
\mathcal{S} \neq \varnothing, \quad \tau^{*}:=\sup \mathcal{S}
$$

If $\tau^{*}=\infty$, then we are done. So we assume the contrary holds, i.e.,

$$
\tau^{*}<\infty
$$

Then, we have

$$
\text { either }\left|\theta\left(\tau^{*}\right)-\theta_{c}\left(\tau^{*}\right)\right|=\varepsilon_{0} \quad \text { or } \quad\left|\theta_{c}\left(\tau^{*}\right)-\theta_{c}^{0}\right|=\varepsilon_{0} .
$$

Next, we will show that the above two cases lead to contradictions, and we conclude $\tau^{*}=\infty$.

Case A. Suppose that $\left|\theta\left(\tau^{*}\right)-\theta_{\mathcal{c}}\left(\tau^{*}\right)\right|=\varepsilon_{0}$. In this case, we can repeat the proof of Proposition 5.2 to get

$$
\varepsilon_{0}^{2}=\left|\theta\left(\tau^{*}\right)-\theta_{c}\left(\tau^{*}\right)\right|^{2}<\frac{\varepsilon_{0}^{2}}{2} e^{-\frac{\kappa_{1} \psi(R)}{12\left(\theta_{c}^{0}+2 \varepsilon_{0}\right)} \tau^{*}} \leq \frac{\varepsilon_{0}^{2}}{2},
$$

which yields a contradiction.

Case B. If there exists $\tau^{*}<\infty$ such that

$$
\left|\theta_{\mathcal{c}}\left(\tau^{*}\right)-\theta_{c}^{0}\right|=\varepsilon_{0}
$$

From Remark 2.2, one has

$$
\theta_{\mathcal{C}}(t)=\theta_{\mathcal{C}}(0)+\frac{1}{2}\left|v_{\mathcal{C}}(0)\right|^{2}+\frac{1}{2} \int_{\Omega}\left|v-v_{\mathcal{C}}(0)\right|^{2} f^{0} d z-\frac{1}{2}\left|v_{\mathcal{C}}(t)\right|^{2}-\frac{1}{2} \int_{\Omega}\left|v-v_{\mathcal{C}}(t)\right|^{2} f d z .
$$

This yields

$$
\begin{aligned}
& \theta_{c}\left(t^{*}\right) \geq \theta_{c}(0)-\frac{1}{2}\left|v_{c}\right|^{2}-\frac{1}{2} \int_{\Omega}\left|v-v_{c}\right|^{2} f d z \geq \theta_{c}^{0}-\left(\left|v_{c}(0)\right|^{2}+\left|x_{c}(0)\right|^{2}\right)-\frac{R^{2}}{8}>\theta_{c}^{0}-\varepsilon_{0} \\
& \theta_{c}\left(t^{*}\right) \leq \theta_{c}(0)+\frac{1}{2}\left|v_{c}(0)\right|^{2}+\frac{1}{2} \int_{\Omega}\left|v-v_{c}(0)\right|^{2} f^{0} d z \leq \theta_{c}^{0}+\frac{1}{2}\left|v_{c}(0)\right|^{2}+\frac{R^{2}}{32 K}<\theta_{c}^{0}+\varepsilon_{0}
\end{aligned}
$$


where we used the relations (8) in (3.6)

$$
\left|v_{\mathcal{c}}(0)\right|^{2}+\left|x_{c}(0)\right|^{2}+\frac{R^{2}}{8}<\varepsilon_{0}, \quad \frac{1}{2}\left|v_{\mathcal{c}}(0)\right|^{2}+\frac{R^{2}}{32 K}<\varepsilon_{0}
$$

Thus we have

$$
\theta_{c}^{0}-\varepsilon_{0}<\theta_{c}\left(t^{*}\right)<\theta_{c}^{0}+\varepsilon_{0},
$$

which yields a contradiction. Therefore for $t \in[0, \infty)$, we have

$$
\left|\theta(t)-\theta_{c}(t)\right|<\varepsilon_{0} \quad \text { for } \quad \theta \in \cup_{t \geq 0} \Omega_{\theta}(f, t), \quad \Omega_{\theta}(f, t) \subseteq\left(\theta_{c}^{0}-2 \varepsilon_{0}, \theta_{c}^{0}+2 \varepsilon_{0}\right) .
$$

Thus, it follows from Propositions 4.2 and 5.2 that the relations

$$
\left|x-x_{c}(t)\right|<\frac{R}{2} e^{-\frac{1}{24} \frac{\kappa_{1} \psi(R)}{\left(\theta_{c}^{0}+2 \varepsilon_{0}\right)} t}, \quad\left|v-v_{c}(t)\right|<\frac{R}{2} e^{-\frac{1}{24} \frac{\kappa_{1} \psi(R)}{\left(\theta_{c}^{0}+2 \varepsilon_{0}\right)} t}, \quad\left|\theta-\theta_{c}(t)\right|<\frac{\varepsilon_{0}}{2} e^{-\frac{\kappa_{1} \psi(R)}{24\left(\theta_{c}^{0}+2 \varepsilon_{0}\right)} t}
$$

imply

$$
d_{x}[f] \leq 2\left|x-x_{c}\right| \leq R e^{-\frac{\kappa_{1} \psi(R)}{24\left(\theta_{c}^{0}+2 \varepsilon_{0}\right)} t}, \quad d_{v}[f] \leq R e^{-\frac{\kappa_{1} \psi(R)}{24\left(\theta_{c}^{0}+2 \varepsilon_{0}\right)} t}, \quad d_{\theta}[f] \leq \varepsilon_{0} e^{-\frac{\kappa_{1} \psi(R)}{24\left(\theta_{c}^{0}+2 \varepsilon_{0}\right)} t},
$$

and we complete the proof of main theorem.

\section{Conclusion}

In this paper, we have studied the asymptotic formation of periodically rotating one-point cluster for the kinetic TCS model in a harmonic potential field. Specifically, we provide a sufficient framework leading to the exponential relaxations of position, velocity and temperature toward one-point cluster. The framework is formulated in terms of well-prepared initial configuration, communication weight functions and coupling strengths. For the full kinetic TCS model, to guarantee the positivity of temperature variables in the whole-time interval, we first make an ansatz so that the support of the kinetic density in temperature variable is always away from zero in some short-time interval. Then we get the local existence of the solution for the kinetic TCS model, and derive the exponential decay properties for the fluctuations of position and velocity for the characteristic system via the dissipative differential inequality in the same small-time interval. Using this result, one can deduce that fluctuations of temperature decays exponentially to zero which improves the ansatz. By the continuity argument, we derived uniform bounds and exponential decay properties of position, velocity and temperature-diameter functionals in the whole-time interval. We also provided a global well-posedness of classical solutions for the full kinetic TCS model by the uniform bound of the kinetic density in any finite-time interval in the Appendix. 


\section{Appendix A. A unique global solvability of classical solution}

In this appendix, we briefly discuss a unique global solvability of smooth solution to the kinetic TCS model.

Note that the local existence of classical solution will be followed from the standard fixed point argument. Thus, we focus on a priori $C^{1}$-bound of $f$ to conclude a global existence of classical solution for the kinetic TCS model.

Theorem A.1. Suppose the framework $\left(\mathcal{F}_{A}\right)-\left(\mathcal{F}_{C}\right)$ holds, and the initial datum $f^{0}$ is $C^{2}$-regular and bounded

$$
\sum_{0 \leq|\alpha|+|\beta|+|\gamma| \leq 2}\left\|\nabla_{x}^{\alpha} \nabla_{v}^{\beta} \partial_{\theta}^{\gamma} f^{0}\right\|_{L_{x, v, \theta}^{\infty}<\infty .}<\infty
$$

Then, for any $T \in(0, \infty)$, there exists a unique classical solution $f \in C^{1}([0, T) \times \Omega)$ to (1.3).

Proof. To get the desired estimate, it suffices to derive $W^{2, \infty}$-estimate of $f$. For this, we only verify $W^{1, \infty}$-estimate of $f$, and similarly we can derive $W^{2, \infty}$-estimate as well after tedious calculations. First, we represent Eq. (1.3) in terms of the nonlinear transport operator

$$
\mathcal{T}:=\partial_{t}+v \cdot \nabla_{x}+\mathcal{F}[f] \cdot \nabla_{v}+\mathcal{G}[f] \partial_{\theta}
$$

Then, we have

$$
\begin{aligned}
\mathcal{T}(f)=- & f \nabla_{v} \cdot \mathcal{F}[f]-f \partial_{\theta} \mathcal{G}[f], \\
\mathcal{T}\left(\partial_{x_{i}} f\right)= & -\partial_{x_{i}} \mathcal{F}[f] \cdot \nabla_{v} f-\partial_{x_{i}} \mathcal{G}[f] \partial_{\theta} f-\nabla_{v} \cdot \partial_{x_{i}} \mathcal{F}[f] f \\
& -\nabla_{v} \cdot \mathcal{F}[f] \partial_{x_{i}} f-\partial_{\theta} \partial_{x_{i}} \mathcal{G}[f] f-\partial_{\theta} \mathcal{G}[f] \partial_{x_{i}} f, \\
\mathcal{T}\left(\partial_{v_{i}} f\right)= & -\partial_{x_{i}} f-\partial_{v_{i}} \mathcal{F}[f] \cdot \nabla_{v} f-\nabla_{v} \cdot \mathcal{F}[f] \partial_{v_{i}} f-\partial_{\theta} \mathcal{G}[f] \partial_{v_{i}} f, \\
\mathcal{T}\left(\partial_{\theta} f\right)= & -\partial_{\theta} \mathcal{F}[f] \cdot \nabla_{v} f-2 \partial_{\theta} \mathcal{G}[f] \partial_{\theta} f-\partial_{\theta} \nabla_{v} \cdot \mathcal{F}[f] f \\
& -\nabla_{v} \cdot \mathcal{F}[f] \partial_{\theta} f-\partial_{\theta}^{2} \mathcal{G}[f] f .
\end{aligned}
$$

Moreover, one has

$$
\begin{aligned}
& \left|\nabla_{v} \cdot \mathcal{F}[f]\right| \leq \frac{\kappa_{1} d}{\theta_{c}^{0}-2 \varepsilon_{0}}, \quad\left|\partial_{x_{i}} \mathcal{F}[f]\right| \leq \frac{\kappa_{1}\|\nabla \psi\|_{L^{\infty} R}}{\theta_{c}^{0}-2 \varepsilon_{0}}+1 \\
& \left|\partial_{\theta} \mathcal{G}[f]\right| \leq \frac{\kappa_{2}}{\left(\theta_{c}^{0}-2 \varepsilon_{0}\right)^{2}}+\frac{\kappa_{1} R}{2\left(\theta_{c}^{0}-2 \varepsilon_{0}\right)^{2}}
\end{aligned}
$$




$$
\begin{aligned}
& \left|\partial_{x_{i}} \mathcal{G}[f]\right| \leq \frac{2 \kappa_{2}\|\nabla \zeta(0)\|_{L^{\infty}}}{\theta_{c}^{0}-2 \varepsilon_{0}}+\frac{\kappa_{1}\|\nabla \psi\|_{L^{\infty}} R^{2}}{2\left(\theta_{c}^{0}-2 \varepsilon_{0}\right)}+R, \\
& \left|\nabla_{v} \cdot \partial_{x_{i}} \mathcal{F}[f]\right| \leq \frac{\kappa_{1} d\|\nabla \psi\|_{L^{\infty}}}{\theta_{c}^{0}-2 \varepsilon_{0}}, \quad\left|\partial_{\theta} \mathcal{F}[f]\right| \leq \frac{\kappa_{1} R}{2\left(\theta_{c}^{0}-2 \varepsilon_{0}\right)^{2}} \\
& \left|\partial_{\theta} \partial_{x_{i}} \mathcal{G}[f]\right| \leq \frac{\kappa_{2}\|\nabla \zeta(0)\|_{L^{\infty}}}{\left(\theta_{c}^{0}-2 \varepsilon_{0}\right)^{2}}+\frac{\kappa_{1}\|\nabla \psi(0)\|_{L^{\infty} R^{2}}}{2\left(\theta_{c}^{0}-2 \varepsilon_{0}\right)^{2}}, \\
& \left|\partial_{\theta} \nabla_{v} \cdot \mathcal{F}[f]\right| \leq \frac{\kappa_{1} d}{\left(\theta_{c}^{0}-2 \varepsilon_{0}\right)^{2}}, \quad\left|\partial_{\theta}^{2} \mathcal{G}[f]\right| \leq \frac{\kappa_{2}}{\left(\theta_{c}^{0}-2 \varepsilon_{0}\right)^{3}}+\frac{\kappa_{1} R^{2}}{2\left(\theta_{c}^{0}-2 \varepsilon_{0}\right)^{3}} .
\end{aligned}
$$

Now we combine (A.1) and (A.2) together and claim that there exists a positive constant $C$ depending only on the communication weight functions ( $\psi$ and $\zeta)$, coupling strengths $\left(\kappa_{1}\right.$ and $\left.\kappa_{2}\right)$ and initial datum $f^{0}$, such that

$$
\mathcal{T}\left(\sum_{0 \leq|\alpha|+|\beta|+|\gamma| \leq 1} \nabla_{x}^{\alpha} \nabla_{v}^{\beta} \partial_{\theta}^{\gamma} f\right) \leq C \sum_{0 \leq|\alpha|+|\beta|+|\gamma| \leq 1} \nabla_{x}^{\alpha} \nabla_{v}^{\beta} \partial_{\theta}^{\gamma} f .
$$

Define the functional $\mathscr{F}(t)$ measuring the $W^{1, \infty}$-norm of $f(\cdot, t)$

$$
\mathscr{F}(t):=\sum_{0 \leq|\alpha|+|\beta|+|\gamma| \leq 1}\left\|\nabla_{x}^{\alpha} \nabla_{v}^{\beta} \partial_{\theta}^{\gamma} f\right\|_{L_{x, v, \theta}^{\infty}}
$$

Then the differential inequality (A.3) implies that along the characteristic (4.10), one has

$$
\frac{d}{d t} \mathscr{F}(t) \leq C \mathscr{F}(t)
$$

This yields

$$
\mathscr{F}(t) \leq \mathscr{F}(0) e^{C t}, \quad t>0 .
$$

Equipped with this a priori $W^{1, \infty}$-norm estimate, standard continuation principle yields a global extension of a local classical solution. The uniqueness part can be done by deriving similar estimate like (A.3) for the difference of two solutions using the a priori bounds (A.4).

\section{Acknowledgments}

The authors would like to thank the anonymous referees for their substantial comments on this work.

The work of S.-Y. Ha is supported by the National Research Foundation of Korea (Grant NRF-2020R1A2C3A01003881), and the work of L. Du is supported by 
the Natural Science Foundation of China (Grants 12001097, 11861131003) and by the China Scholarship Council. This work was completed while the first author visited HYKE-Hwarang Lab, Seoul National University. She would like to thank Professor Ha for his hospitality during the academic year of 2020.

\section{References}

[1] J. A. Acebron, L. L. Bonilla, C. J. Pérez Vicente, F. Ritort, and R. Spigler, The Kuramoto model: A simple paradigm for synchronization phenomena, Rev. Mod. Phys. 77 (2005), 137-185.

[2] G. Albi, N. Bellomo, L. Fermo, S.-Y. Ha, J. Kim, L. Pareschi, D. Poyato, and J. Soler, Vehicular traffic, crowds, and swarms: from kinetic theory and multiscale methods to applications and research perspectives, Math. Models Methods Appl. Sci. 29 (2019), 1901-2005.

[3] J. Buck, and E. Buck, Biology of synchronous flashing of fireflies, Nature 211 (1966), 562-564.

[4] J. A. Cañizo, J. A. Carrillo, and J. Rosado, A well-posedness theory in measures for some kinetic models of collective motion, Math. Models Methods Appl. Sci. 21 (2011), 515-539.

[5] J. A. Carrillo, M. R. D'Orsogna, and V. Panferov, Double milling in self-propelled swarms from kinetic theory, Kinetic and Related Models 2 (2009), 363-378.

[6] J. A. Carrillo, M. Fornasier, J. Rosado, and G. Toscani, Asymptotic flocking dynamics for the kinetic Cucker-Smale model, SIAM J. Math. Anal. 42 (2010), 218-236.

[7] H. Cho, L. Du, and S.-Y. Ha, Emergence of periodically rotating one-point clusters in thermodynamic Cucker-Smale ensemble, arXiv:2110.11787, 2021.

[8] Y.-P. Choi, Large-time behavior for the Vlasov/compressible Navier-Stokes equations, J. Math. Phys. 57 (2016), 071501.

[9] Y.-P. Choi, S.-Y. Ha, J. Jung, and J. Kim, Global dynamics of the thermomechanical Cucker-Smale ensemble immersed in incompressible viscous fluids, Nonlinearity 32 (2019), 159-1640.

[10] Y.-P. Choi, S.-Y. Ha, J. Jung, and J. Kim, On the coupling of kinetic thermomechanical Cucker-Smale equation and compressible viscous fluid system, J. Math. Fluid Mech. 22 (2020), 4.

[11] P. Degond, and S. Motsch, Large-scale dynamics of the Persistent Turning Walker model of fish behavior, J. Stat. Phys. 131 (2008), 989-1022.

[12] R. Duan, M. Fornasier, and G. Toscani, A kinetic flocking model with diffusion, Commun. Math. Phys. 300 (2010), 95-145.

[13] S.-Y. Ha, T. Ha, and J. Kim, Asymptotic dynamics for the Cucker-Smale-type model with the Rayleigh friction, J. Phys. A: Math. Theor. 43 (2010), 315201. 
[14] S.-Y. Ha, J. Kim, C. Min, T. Ruggeri, and X. Zhang, Uniform stability and meanfield limit of a thermodynamical Cucker-Smale model, Quart. Appl. Math. 2 (2019), 131-176.

[15] S.-Y. Ha, J. Kim, and T. Ruggeri, Emergent behaviors of thermodynamically consistent particle, SIAM J. Math. Anal. 30 (2018), 3092-3121.

[16] S.-Y. Ha, J. Kim, and X. Zhang, Uniform stability of the Cucker-Smale model and its application to the mean-fielld limit, Kinet. Relat. Models 11 (2018), 1157-1181.

[17] S.-Y. Ha, and J. G. Liu, A simple proof of the Cucker-Smale flocking dynamics and mean-fileld limit, Commun. Math. Sci. 7 (2009), 297-325.

[18] S.-Y. Ha, and T. Ruggeri, Emergent dynamics of a thermodynamically consistent particle model, Arch. Ration. Mech. Anal. 223 (2017), 1397-1425.

[19] S.-Y. Ha, and E. Tadmor, From particle to kinetic and hydrodynamic descriptions of flocking, Kinet. Relat. Models 1 (2008), 415-435.

[20] S. Motsch, and E. Tadmor, Heterophilious dynamics enhances consensus, SIAM Rev. 56 (2014), 577-621.

[21] C. S. Peskin, Mathematical Aspects of Heart Physiology, Courant Institute of Mathematical Sciences, 1975.

[22] A. Pikovsky, M. Rosenblum, and J. Kurths, Synchronization: A Universal Concept in Nonlinear Sciences, Cambridge University Press, 2001.

[23] R. Shu, and E. Tadmor, Flocking hydrodynamics with external potentials, Arch Rational Mech. Anal. 238 (2020), 347-381.

[24] J. Toner, and Y. Tu, Flocks, herds, and Schools: A quantitative theory of flocking, Physical Review E 58 (1998), 4828-4858.

[25] C. M. Topaz, and A. L. Bertozzi, Swarming patterns in a two-dimensional kinematic model for biological groups, SIAM J. Appl. Math. 65 (2004), 152-174.

[26] T. Vicsek, and A. Zefeiris, Collective motion, Phys. Rep. 517 (2012), 71-140.

[27] A. T. Winfree, The Geometry of Biological Time, Springer, 1980. 